\title{
The flat phase of quantum polymerized membranes
}

\author{
O. Coquand ${ }^{1, *}$ and D. Mouhanna ${ }^{1, \dagger}$ \\ ${ }^{1}$ Sorbonne Universités, UPMC Univ Paris 06, LPTMC, CNRS UMR 7600, F-75005, Paris, France
}

\begin{abstract}
We investigate the flat phase of quantum polymerized phantom membranes by means of a nonperturbative renormalization group approach. We first implement this formalism for general quantum polymerized membranes and derive the flow equations that encompass both quantum and thermal fluctuations. We then deduce and analyze the flow equations relevant to study the flat phase and discuss their salient features : quantum to classical crossover and, in each of these regimes, strong to weak coupling crossover. We finally illustrate these features in the context of free standing graphene physics.
\end{abstract}

PACS numbers: 87.16.D-,11.10.Hi, 11.15.Tk

\section{INTRODUCTION}

The discovery of graphene [1,2] has generated an almost unprecedented activity devoted to understanding its exceptional mechanical, optical and electronic properties including high electronic mobility, mechanical strength, optical transmittance and thermal conductivity (see, e.g., [3] and references therein). From the mechanical point of view graphene appears, due to its one-atomthick layer structure and its elastic properties, as the first realization of a perfect two dimensional (2D) crystalline - or polymerized - membrane (see, e.g., [4]). From the electronic point of view graphene displays a low-energy spectrum made of massless chiral Dirac fermions that mimic quantum electrodynamics [4]. Also one of the most striking features of graphene and graphene-like materials is the interplay between the elastic and electronic degrees of freedom. For instance elastic distortions of graphene layers modify the electronic band structure and thus the conductivity of this material [4-10]. Reciprocally electronic degrees of freedom have been proposed to be at the origin of ripples formation [11-14]. This situation has opened a very promising road for the design of graphene and graphene-like materials with optimized optical and electronic properties by means of "strain engineering" (see [15] and references therein). From a more fundamental point of view the specific interaction between the elastic and electronic degrees of freedom in graphene has been at the origin of a very challenging many-body problem combining both high-energy and condensed matter physics concepts that has notably received an elegant and fruitful formalization in terms of gauge-field theory and field theory - Dirac equation - in curved space (see $[9,16]$ for reviews). While much progress has been realized toward an understanding of the full problem combining electronic and elastic degrees of freedom, a complete and quantitatively well controlled description of the mechanical part of the problem, that is already associated with

\footnotetext{
*Electronic address: coquand@lptmc.jussieu.fr

$\dagger$ Electronic address: mouhanna@lptmc.jussieu.fr
}

a non-trivial interacting field theory, is still lacking.

From this point of view the study of the joint effects of interactions and fluctuations on the long distance properties of 2D classical polymerized membranes is already a well explored subject (see [17] for a state of the art). As revealed by early studies [18-22] the most important physical effect taking place in these systems is the stabilization of long-range orientational order through the anharmonic coupling between stretching and bending degrees of freedom. Indeed the coupling between the corresponding in-plane, $\mathbf{u}$, and out-of-plane, $\mathbf{h}$, modes leads to a drastic reduction of the fluctuations of the latter that are otherwise, i.e. at the harmonic level, divergent. Technically this can be understood through the appearance of a momentum dependent effective bending rigidity modulus $\kappa(q) \sim q^{-\eta}$ [18], where $\eta$ is a positive exponent, whose increase at low momenta enforces the rigidity and thus the stability of the membrane. The exponent $\eta$ has been computed by means of analytical computations on the continuous effective theory of polymerized membranes and by means of Monte Carlo and molecular dynamics simulations on discretized versions of membranes or on models of graphene using empirical potentials (see [16] for a review). Although a significant dispersion of the results is observed the agreement between the analytical predictions $-\eta \in[0.789-0.85]-$ and numerical computations $-\eta \in[0.72-0.85]$ - leaves little doubt as for the adequacy of continuous models of polymerized membranes to describe the long distance physics of membranes as well as the elastic degrees of freedom of graphene (see for instance [23]).

Being given the importance of the interplay between anharmonicities and thermal fluctuations regarding the question of stability and, more generally, the long distance behaviour, of membranes, a question that naturally arises is that of the joint role of anharmonicities and quantum fluctuations in these systems. This question is not purely formal since simple estimate [24] as well as computations by means of a self-consistent field theoretical approach using a interatomic potential specific to graphene [25], have shown that, for this material, the typical temperature up to which quantum effects are significant should lie around $T \sim 1000 \mathrm{~K}$. Several recent 
works have been devoted to understanding the effects induced by quantum fluctuations on membranes (see [16] for a review). In [12-14] studies including both anharmonic elastic contributions and electron-phonon coupling have been performed using the Self-Consistent Screening Approximation (SCSA), extending the approaches performed for classical membranes [22, 26, 27]. In these works it has been shown that the presence of a strong coupling between electronic and elastic degrees of freedom leads to a drastic decrease of the bending rigidity inducing an instability in the flexural sector. From the Renormalization Group ( $\mathrm{RG}$ ) point of view this instability is reflected in the appearance of a critical point characterizing a rippling transition. In the opposite limit of vanishing electron-phonon coupling, the RG equations for the Young modulus $[12,13]$ and bending rigidity modulus [12] describe a (logarithmic) decrease of the former and a (logarithmic) increase of the latter, leading to a stable configuration for the membrane, as in the classical case. In [28] a RG approach of quantum membranes has been performed in a perturbative context, leading to the opposite - and surprising - result that quantum fluctuations induce the phenomenon of ultra-violet asymptotic freedom, and thus a strong coupling behaviour in the infra-red. According to [28] this would lead to a destabilization of the flat phase at long distances. Finally in [24] the thermodynamics of membranes at very low temperatures has been explored through a computation of the self-energy of the flexural mode at one-loop and then in a self-consistent way in the spirit of the early approach to classical membranes of Nelson and Peliti [18]. The aim of this approach, that we refer to as Self-Consistent Born Approximation (SCBA) - to be distinguished from the SCSA by the fact that only the flexural mode propagator, and thus not the interaction, is treated self-consistently - was to take into account both anharmonic and retardation interaction terms neglected in previous approaches $[13,28]$. The main and, again, surprising result of this approach is the existence, at vanishing temperature, of a power-law momentum scaling of the bending rigidity modulus $\kappa(q) \sim q^{-\eta}$ with a critical exponent $\eta=2$, as opposed to the logarithmic behaviour obtained in previous approaches [12-14, 28]. This result has been the subject of a controversy as for the interpretation of the corrections to the self-energy obtained in this work (see $[29,30]$ for details).

In this article we develop an approach to quantum polymerized membranes realized within a Nonperturbative Renormalization Group (NPRG) framework, the effective average action method [31-39], extending previous works performed on classical membranes [40-44] to their quantum counterpart. We first derive the general $\mathrm{RG}$ equations for a model of membranes that describes both the phonon and flexural modes and encompasses both quantum and classical, i.e. thermal, fluctuations. We then focus on the flat phase of membranes whose RG equations are obtained from the latter via the phenomenon of decoupling of phonon modes. At vanishing temperature, the analysis of the equations shows that the long distance behaviour of membranes is controlled by a trivial - gaussian - fixed point in agreement with previous work performed within the SCSA framework [12-14] but in disagreement with the results found within a recent perturbative approach in which an ultra-violet asymptotic freedom is predicted [28] and with those obtained within a recent SCBA approach leading to a non-trivial critical exponent $\eta$ [24]. At any finite temperature the RG flow drives the system toward an effective high temperature regime which is well described by the classical theory of membranes that predicts a long distance behaviour governed by a non-trivial infra-red fixed point. This crossover between the short distance - quantum regime and long-distance - classical - regime as well as the crossovers between weak and strong coupling regimes are discussed within the context of free standing graphene physics.

Our article is organized as follows. In Section II we present the action used to investigate the long distance behaviour of quantum polymerized membranes and discuss the fluctuations around the flat phase. In section III we describe the effective average action method employed to derive the NPRG equations for quantum membranes. In section IV we derive the RG flow for general membranes and, then, derive and analyze that relevant to study their flat phase, at finite, at vanishing and at high temperatures, and compare our results with previous works. In section $\mathrm{V}$ we illustrate our findings in the context of the physics of free standing graphene. Finally, in section VI, we conclude.

\section{THE ACTION AND FLUCTUATIONS AROUND THE FLAT PHASE}

\section{A. The action for quantum membranes}

We consider a $D$-dimensional quantum membrane embedded in a $d$-dimensional Euclidean space. Each point of the membrane is parametrized by $D$ internal coordinates $\mathbf{x} \equiv x_{\gamma}, \gamma=1 \ldots D$. Its location in the Euclidean space is realized through the embedding: $\mathbf{x} \rightarrow \mathbf{R}(\mathbf{x}, \tau)$ where $\mathbf{R}$ is a $d$-component vector field with components $R_{i}, i=1 \ldots d$ and $\tau$ an imaginary time (see, e.g., [45]). The action of the model we consider is given by:

$$
\begin{aligned}
S & {[\mathbf{R}]=\int_{0}^{\beta} d \tau \int d^{D} x\left\{\frac{\rho}{2}\left(\partial_{\tau} \mathbf{R}\right)^{2}+\frac{\kappa}{2}\left(\partial_{\gamma} \partial_{\gamma} \mathbf{R}\right)^{2}\right.} \\
& \left.+\frac{\tilde{\mu}}{4}\left(\partial_{\gamma} \mathbf{R} . \partial_{\nu} \mathbf{R}-\tilde{\zeta}^{2} \delta_{\gamma, \nu}\right)^{2}+\frac{\tilde{\lambda}}{8}\left(\partial_{\gamma} \mathbf{R} . \partial_{\gamma} \mathbf{R}-D \tilde{\zeta}^{2}\right)^{2}\right\}
\end{aligned}
$$

where $\tau$ takes its values in $[0, \beta]$ with $\beta=1 / T$ ( $\hbar$ and $k_{B}$ are taken equal to 1 ). In Eq.(1) the first term represents the kinetic part of the action, the second one a bending energy term while the other ones correspond to harmonic and anharmonic elastic terms. The stability of the action 
implies that $\tilde{\mu}$ and the bulk modulus $2 \tilde{\mu} / D+\tilde{\lambda}$ must be positive. The action (1) is the extension to the quantum case of the action used to study classical polymerized membranes. At mean-field level, varying the squared extension factor $\tilde{\zeta}^{2}$, it describes a phase transition between a crumpled phase for $\tilde{\zeta}^{2}<0$, with a vanishing average value of the tangent vectors $\left\langle\partial_{\gamma} \mathbf{R}\right\rangle$, and a flat phase for $\tilde{\zeta}^{2}>0$ defined by:

$$
\langle\mathbf{R}(\mathbf{x}, \tau)\rangle=\tilde{\zeta} \sum_{\alpha=1}^{D} x_{\alpha} \mathbf{e}_{\alpha}
$$

that implies $\left\langle\partial_{\gamma} \mathbf{R}\right\rangle=\tilde{\zeta} \mathbf{e}_{\gamma}$ with $\gamma=1 \ldots D$, the $\mathbf{e}_{\gamma}$ 's forming an orthonormal set of $D$ vectors that generate the flat configuration (2).

The form (1), in which the action is expanded around the flat configuration (2), is particularly well-suited to study the flat phase of membranes we are interested in. It is, in particular, reminiscent of the action used to study perturbatively this phase if one replaces, in Eq.(1), the strain tensor $u_{\gamma \nu}=\partial_{\gamma} \mathbf{R} . \partial_{\nu} \mathbf{R}-\tilde{\zeta}^{2} \delta_{\gamma, \nu}$ by its expansion in terms of in-plane phonon modes $u_{\gamma}$ and out-of-plane flexural modes $\mathbf{h}: u_{\gamma \nu}=\frac{1}{2}\left(\partial_{\gamma} u_{\nu}+\partial_{\nu} u_{\gamma}+\partial_{\gamma} \mathbf{h} . \partial_{\nu} \mathbf{h}+\right.$ $\left.\partial_{\gamma} u_{\sigma} \partial_{\nu} u_{\sigma}\right)$. However we emphasize the fact that, within our approach, we do not use this explicit decomposition in terms of $\mathbf{u}$ and $\mathbf{h}$ fields. Indeed we keep a full rotationally invariant formalism during the whole computation where only the field $\mathbf{R}$ - and its derivatives - come into play. This is possible since our approach being nonperturbative, we are not restricted to study weak coupling constants, weak fluctuations regimes. One is able, in particular, to investigate both the crumpling-to-flat transition and the flat phase using the same action, as done in [40-44], while the corresponding fixed points are nonperturbatively connected to each other. Technically this implies, for instance, that one can, and must, keep the quadratic term in the phonon field $\mathbf{u}$ in the expression of $u_{\gamma \nu}$ above that is generally discarded.

\section{B. Spectrum and fluctuations}

One can compute, from Eq.(1), the propagator, and thus the spectrum of excitations in the flat phase. The propagator is given by the second derivative of $S[\mathbf{R}]$ with respect to the field $\mathbf{R}$, taken in the configuration Eq.(2). In Fourier space the (imaginary) time direction is compact and, choosing periodic boundary conditions in time $\mathbf{R}(\mathbf{x}, \tau=0)=\mathbf{R}(\mathbf{x}, \tau=\beta)$, the field $\mathbf{R}(\mathbf{x}, \tau)$ can be expanded according to:

$$
\mathbf{R}(\mathbf{x}, \tau)=\frac{1}{\beta} \sum_{\omega_{n}} \int_{q} \mathbf{R}\left(\mathbf{q}, \omega_{n}\right) e^{i\left(\mathbf{q} \cdot \mathbf{x}-\omega_{n} \tau\right)}
$$

where $\int_{q}$ stands for $\int d^{D} q /(2 \pi)^{D}$ and the $\omega_{n}$ are Matsubara frequencies: $\omega_{n}=2 \pi n / \beta, n \in \mathbb{Z}$. The resulting propagator splits into three parts:

$$
\left\{\begin{array}{l}
G_{F}^{-1}\left(\mathbf{q}, \omega_{n}\right)=\kappa q^{4}+\rho \omega_{n}^{2} \\
G_{\|}^{-1}\left(\mathbf{q}, \omega_{n}\right)=\kappa\left(q^{4}+\widetilde{m}_{2}^{2} q^{2}\right)+\rho \omega_{n}^{2} \\
G_{\perp}^{-1}\left(\mathbf{q}, \omega_{n}\right)=\kappa\left(q^{4}+\widetilde{m}_{1}^{2} q^{2}\right)+\rho \omega_{n}^{2}
\end{array}\right.
$$

with $\widetilde{m}_{1}^{2}=\tilde{\mu} \tilde{\zeta}^{2} \kappa^{-1}$ and $\widetilde{m}_{2}^{2}=(2 \tilde{\mu}+\tilde{\lambda}) \tilde{\zeta}^{2} \kappa^{-1}$ and where $q=|\mathbf{q}|$. In Eqs. $(4 \mathrm{a})-(4 \mathrm{c}), G_{F}^{-1}\left(\mathbf{q}, \omega_{n}\right)$ is associated with $d-D$ flexural modes; $G_{\perp}^{-1}\left(\mathbf{q}, \omega_{n}\right)$ and $G_{\|}^{-1}\left(\mathbf{q}, \omega_{n}\right)$ are associated respectively with the $D-1$ transverse (directed orthogonally to $\mathbf{q}$ ) and a single longitudinal (directed along q) phonon modes. We have also introduced two squared masses $\widetilde{m}_{1}^{2}$ and $\widetilde{m}_{2}^{2}$ in the propagators of the transverse and longitudinal phonon modes. This rather unusual way to represent these latter modes as a kind of "massive" flexural modes will take its full meaning in the context of the NPRG approach below where masses acquire a dynamical, running, status.

The expressions (4a)-(4c) can be used to evaluate the importance of fluctuations in the flat phase. Due to the dispersion relation of flexural modes it appears that the fluctuations associated with these modes are most important; hence we concentrate on them. At high temperatures only the vanishing frequency mode contributes and the propagator of the flexural - $\mathbf{h}$ - modes, at low momenta, is given by the classical expression:

$$
G_{F}\left(\mathbf{q}, \omega_{n}\right) \underset{q \rightarrow 0, T \rightarrow \infty}{\sim} \frac{1}{\kappa q^{4}} .
$$

This allows to compute the fluctuations of the normals to the membrane that characterize the existence or absence of long-range orientational order. Standard computations show that they are essentially given by the spatial average of the correlation function of the $\partial_{\gamma} \mathbf{h}$ field. One finds, at the harmonic level:

$$
\left\langle\left\langle\left(\partial_{\gamma} \mathbf{h}\right)^{2}\right\rangle\right\rangle_{h} \sim \int d^{D} q q^{2} \frac{1}{\kappa q^{4}} \sim L^{2-D}
$$

where $L$ is the typical size of the system and $\langle\langle\ldots\rangle\rangle$ denotes both a thermodynamical and momentum average. One recovers the classical result that the system displays quasi-long range orientational order in two dimensions. This behaviour is modified by anharmonic contributions that induce a $q$-dependence of the bending rigidity modulus, $\kappa(q) \sim q^{-\eta}$ [18], and make the integral

$$
\left\langle\left\langle\left(\partial_{\gamma} \mathbf{h}\right)^{2}\right\rangle\right\rangle_{a n h} \sim \int d^{D} q q^{2} \frac{1}{\kappa(q) q^{4}} \sim L^{2-D-\eta}
$$

convergent in $D=2$ for any positive value of $\eta$.

At very low temperatures, all Matsubara frequencies contribute and they can be considered as continuous. One thus has:

$$
G_{F}\left(\mathbf{q}, \omega_{n}\right) \underset{q \rightarrow 0, T \rightarrow 0}{\sim} \frac{1}{\kappa q^{4}+\rho \omega^{2}}
$$


so that :

$$
\left\langle\left\langle\left(\partial_{\gamma} \mathbf{h}\right)^{2}\right\rangle\right\rangle_{h} \sim \int d^{D} q d \omega \frac{q^{2}}{\kappa q^{4}+\rho \omega^{2}} \sim L^{-D}
$$

which converges in particular in $D=2$. This means that quantum fluctuations are not strong enough to destabilize the long-range orientational order unless interactions change this behaviour.

\section{THE EFFECTIVE AVERAGE ACTION METHOD}

We now present the effective average action method that we use to compute the effects of interactions in quantum membranes.

\section{A. General principle}

The effective average action method (see [31] and [3239] for reviews) achieves, at the level of the (Gibbs) free energy, the Wilson-Kadanoff bloc-spin program. It involves a central object, $\Gamma_{k},-k$ being a running scale that is essentially a running Gibbs free energy where only fluctuations of momenta $q \geq k$ have been integrated out. Note that we consider, for the time being, a classical situation and thus a classical free energy. At the microscopic lattice scale $\Lambda$, no fluctuation has been integrated out and $\Gamma_{k=\Lambda}$ identifies with the microscopic action $S$ while, at long distances, i.e. at $k=0$, it identifies with the standard Gibbs free energy $\Gamma$ :

$$
\left\{\begin{array}{l}
\Gamma_{k=\Lambda}=S \\
\Gamma_{k=0}=\Gamma
\end{array}\right.
$$

and, at any finite momentum scale $0<k<\Lambda, \Gamma_{k}$ interpolates smoothly between these two limits. In practice the integration over the high momenta $-q>k$ - modes is realized by adding to the microscopic action, Eq.(1), a $k$-dependent "mass" term :

$$
\Delta S_{k}[\mathbf{R}]=\frac{1}{2} \int_{\mathbf{q}} R_{k, a b}(\mathbf{q}) R_{a}(\mathbf{q}) R_{b}(-\mathbf{q})
$$

so that the partition function, in presence of a source term, reads:

$$
\mathcal{Z}_{k}[\mathbf{J}]=\int \mathcal{D} \mathbf{R} \exp \left(-S[\mathbf{R}]-\Delta S_{k}[\mathbf{R}]+\int_{\mathbf{q}} \mathbf{J}(\mathbf{q}) \cdot \mathbf{R}(-\mathbf{q})\right) .
$$

Note that, in the rest of the article, the function $R_{k, a b}(\mathbf{q})$, discussed below, will be considered as diagonal in field space, i.e. $R_{k, a b}(\mathbf{q})=R_{k}(\mathbf{q}) \delta_{a, b}$.

The role of the $\Delta S_{k}[\mathbf{R}]$ term in Eq.(12) is to "ballast" the low momenta modes and to make that only the high momenta modes are effectively integrated out. To realize this program, and to make sure that $\Gamma_{k}$ meets the conditions Eq.(10a)-(10b), the function $R_{k}(\mathbf{q})$ must obey several constraints: $i$ ) it behaves as $k^{\alpha}$ - with suitable power $\alpha>0$ - at low momenta ii) it vanishes at high momenta:

$$
\left\{\begin{array}{lll}
R_{k}(\mathbf{q}) \sim k^{\alpha} & \text { when } & q \ll k \\
R_{k}(\mathbf{q}) \rightarrow 0 & \text { when } & q \gg k
\end{array}\right.
$$

The relation (13a) implies that the low momenta modes are affected by a "mass" term Eq.(11) with mass $k^{\alpha}$ that prevents their propagation - they "decouple" while the relation (13b) implies that the high momenta modes are kept untouched. These two relations can be translated in terms of the $k$-dependence of $R_{k}(\mathbf{q})$ :

$$
\left\{\begin{array}{lll}
R_{k}(\mathbf{q}) \sim \Lambda^{\alpha} & \text { when } & k \rightarrow \Lambda \\
R_{k}(\mathbf{q}) \rightarrow 0 & \text { when } & k \rightarrow 0 .
\end{array}\right.
$$

The relation (14a) means that, when $k \rightarrow \Lambda, \Delta S_{k}[\mathbf{R}]$ acts as a large mass term for all modes, what implies that almost no mode contributes to the functional integral in Eq.(12); thus no fluctuation has been included. The relation (14b) means that, when $k \rightarrow 0, \Delta S_{k}[\mathbf{R}]$ plays no role, so that all modes contribute to the functional integration; thus all fluctuations have been included. A typical cut-off function satisfying all the previous requirements is given by the " $\Theta$ " cut-off [46]:

$$
R_{k}(\mathbf{q})=Z_{k} q^{\alpha}\left(\left(\frac{k}{q}\right)^{\alpha}-1\right) \Theta\left(1-\frac{q^{2}}{k^{2}}\right)
$$

where $\Theta$ is the step function and $Z_{k}$ is a field renormalization - see below. Note that the extension to a quantum system can lead to consider $R_{k}$ as a function of both momentum $\mathbf{q}$ and frequency $\omega$ - see below. We now define the running Gibbs free energy $\Gamma_{k}$ as a modified Legendre transform of the Helmholtz free energy $W_{k}[\mathbf{J}]=\ln \mathcal{Z}_{k}[\mathbf{J}]$ [32-39]:

$$
\Gamma_{k}[\mathbf{r}]=-W_{k}[\mathbf{J}]+\int_{\mathbf{q}} \mathbf{J}(\mathbf{q}) \cdot \mathbf{r}(-\mathbf{q})-\Delta S_{k}[\mathbf{r}]
$$

where $\mathbf{r}$ is the expectation value of the microscopic field $\mathbf{R}$ in presence of an external source $\mathbf{J}$ :

$$
r_{i}(\mathbf{x})=\left\langle R_{i}(\mathbf{x})\right\rangle=\frac{\delta W_{k}[\mathbf{J}]}{\delta J_{i}(\mathbf{x})}
$$

and $\Delta S_{k}[\mathbf{r}]$ is the macroscopic counterpart of the microscopic mass term Eq.(11).

From Eqs.(16) and (14b) one sees that, when $k \rightarrow 0$, $\Gamma_{k}[\mathbf{r}]$ coincides with the usual free energy $\Gamma$. The opposite limit $k \rightarrow \Lambda$ is a little bit more tricky. From Eq.(16) one has:

$$
J_{i}(\mathbf{q})=\frac{\delta \Gamma_{k}[\mathbf{r}]}{\delta r_{i}(-\mathbf{q})}+R_{k}(\mathbf{q}) r_{i}(\mathbf{q})
$$


Using then Eqs.(12), (16) and (18) one gets:

$$
\begin{aligned}
e^{-\Gamma_{k}[\mathbf{r}]}= & \int \mathcal{D} \mathbf{R} \exp (-S[\mathbf{R}] \\
& \left.+\int_{\mathbf{q}} \frac{\delta \Gamma_{k}[\mathbf{r}]}{\delta r_{i}(\mathbf{q})}\left(R_{i}(\mathbf{q})-r_{i}(\mathbf{q})\right)-\Delta S_{k}[\mathbf{R}-\mathbf{r}]\right)
\end{aligned}
$$

In the limit $k \rightarrow \Lambda, R_{k}(\mathbf{q})$ is, from Eq.(14a), very large - infinite if we assume $\Lambda \rightarrow \infty$ - so that the mass term $\exp \left(-\Delta S_{k}[\mathbf{R}-\mathbf{r}]\right)$ acts essentially as a hard constraint, $\delta(\mathbf{R}-\mathbf{r})$, in the functional integral Eq.(19) [63]. In this way $\Gamma_{k=\Lambda}[\mathbf{r}]$ identifies with the microscopic action $S[\mathbf{r}]$, Eq.(1). We thus fully recover the constraints of Eqs.(10a)-(10b).

\section{B. The Wetterich equation}

Once the limits of $\Gamma_{k}$ have been given it remains to precise its $k$-dependence. It is provided by the Wetterich equation [47]:

$$
\partial_{t} \Gamma_{k}[\mathbf{r}]=\frac{1}{2} \operatorname{Tr}\left\{\partial_{t} R_{k}\left(\Gamma_{k}^{(2)}[\mathbf{r}]+R_{k}\right)^{-1}\right\}
$$

where one defines a RG"time" $t=\ln k / \Lambda$ and where the trace has to be understood as a space (or momentum) $D$ dimensional integral as well as a summation over internal indices. An equivalent and useful expression is given by:

$$
\partial_{t} \Gamma_{k}[\mathbf{r}]=\frac{1}{2} \widehat{\partial}_{t} \operatorname{Tr} \ln \left(\Gamma_{k}^{(2)}[\mathbf{r}]+R_{k}\right)
$$

where $\widehat{\partial}_{t}$ means that the $t$-derivative only acts on $R_{k}$. In Eqs. (20) and (21) $\Gamma_{k}^{(2)}[\mathbf{r}]$ is the inverse propagator, the second derivative of $\Gamma_{k}$ with respect to the field $\mathbf{r}$ :

$$
\Gamma_{k, i j}^{(2)}[\mathbf{r} ; \mathbf{q},-\mathbf{q}]=\frac{\delta^{2} \Gamma_{k}[\mathbf{r}]}{\delta r_{i}(\mathbf{q}) \delta r_{j}(-\mathbf{q})}
$$

taken in a generic field configuration. Physically Eq.(20) describes the evolution of the effective action $\Gamma_{k}$ when $k$ is lowered and when fluctuations at lower and lower momentum scales are taken into account, its dynamics being controlled by the $k$-dependence of $R_{k}, \partial_{t} R_{k}$. The cut-off function $R_{k}$ also enters Eq.(20) as an additive term to the inverse propagator $\Gamma_{k}^{(2)}$ and directly impacts the propagation of the corresponding mode of momentum $q$ in the implicit integral in Eq.(20), what allows to realize the bloc spin program described above.

Technically two properties of $R_{k}$ should be emphasized. First, since $R_{k}(\mathbf{q}) \sim k^{\alpha}$ at low momenta it acts as an effective infra-red regulator for the corresponding modes and prevents the occurrence of infra-red divergences at any finite value of $k$. The exponent $\alpha$ is chosen to realize this aim; it is generally taken equal to 2 in usual, $O(N)$-like, theories, that involve a spatial kinetic term of order $\partial^{2}$. In the case of membranes it is taken equal to 4 since, there, the spatial kinetic term is of order $\partial^{4}$. The regular behaviour that follows allows to investigate the critical physics by progressively lowering $k$ without having recourse to an $\epsilon$-expansion or other similar tools. Second, since $\partial_{t} R_{k}(\mathbf{q})$ contributes essentially for momenta $q \leq k$, as it can be checked directly using the expression Eq.(15), it makes the RG flow ultra-violet finite.

\section{Properties}

We now discuss the main properties of Eq.(20), which have been otherwise extensively described in several reviews [32-39]. First Eq.(20) is an exact equation. It encompasses all perturbative (magnons, phonons, flexurons modes, etc) and nonperturbative (bound states, topological excitations, instantons, etc) features of the underlying theory. Second Eq.(20) involves a single momentum integral and thus displays a one-loop structure. This makes the momentum dependence of computations much simpler than their perturbative counterparts. In practice this one-loop structure allows a direct comparison with the leading order of all perturbative computations: weakcoupling, low-temperature, $1 / N$ or $1 / d$ expansions, etc. As an illustration replacing in Eq. (20) $\Gamma_{k}^{(2)}[\mathbf{r}]$ by the second derivative of the classical action, $S^{(2)}$, leads to the usual one-loop effective action [32]:

$$
\Gamma_{k}[\mathbf{r}]=S[\mathbf{r}]+\frac{1}{2} \operatorname{Tr} \ln \left(S^{(2)}[\mathbf{r}]+R_{k}\right) .
$$

Of course the complexity is hidden elsewhere, precisely in the fact that Eq.(20) involves the full field-dependent propagator $\Gamma_{k}^{(2)}[\mathbf{r}]$. This situation fundamentally differs from that met within the perturbative framework were the propagator is generally considered in a vanishing field configuration. This is, of course, a crucial point of this framework that allows for nonperturbative investigations. Nevertheless this complexity forces us to perform approximations that mainly consist in doing truncations of the effective action $\Gamma_{k}[\mathbf{r}]$. In this way the computations are doable and the nonperturbative character of the method is kept intact as far as the right-hand side of Eq.(20) is not expanded in powers of any usual small parameter as a coupling constant, the temperature, $1 / N$, $1 / d$ etc. Improving the ansatz allows to check the stability of the results and then the adequacy of the truncation used.

An efficient truncation scheme (see [32-39] for discussions concerning truncations) is the derivative expansion where $\Gamma_{k}$ is expanded in powers of the derivatives of the order parameter. It is justified when one focuses on the critical physics, or more generally, on the long distance behaviour of a system and also when this behaviour is controlled by the elementary excitations as opposed to the case where bound states occur. In this last case, as 
in other situations involving for instance fermionic excitations, one has to take into account the whole momentum structure and other kinds of approximations should be used (see for instance [35, 48-50]). The derivative expansion can be - and is often - combined with an expansion in powers of the order parameter itself around a given field configuration, generally the minimum of the effective action. The great benefit of this combined derivative/field expansion is to convert the functional Eq.(20) to a set of differential equations for the coupling constants entering in the ansatz, as in the perturbative context. Reaching accurate results can require to deal with high orders, see for instance [51]. However we insist on the fact that, even at the lowest orders, this expansion provides qualitatively - and also sometimes quantitatively correct results in numerous contexts (see [32-39]). This relies on the very structure of Eq.(20) that, even approximated by means of truncations of the action, remains nonperturbative.

\section{Effective average action for quantum membranes}

In the present work we consider a truncation directly inspired by Eq.(1) that reads:

$$
\begin{gathered}
\Gamma_{k}[\mathbf{r}]=\int_{0}^{\beta} d \tau \int d^{D} x\left\{\frac{Z_{k}^{\tau}}{2}\left(\partial_{\tau} \mathbf{r}\right)^{2}+\frac{Z_{k}}{2}\left(\partial_{\gamma} \partial_{\gamma} \mathbf{r}\right)^{2}\right. \\
\left.+\frac{\mu_{k}}{4}\left(\partial_{\gamma} \mathbf{r} \cdot \partial_{\nu} \mathbf{r}-\zeta_{k}^{2} \delta_{\gamma, \nu}\right)^{2}+\frac{\lambda_{k}}{8}\left(\partial_{\gamma} \mathbf{r} \cdot \partial_{\gamma} \mathbf{r}-D \zeta_{k}^{2}\right)^{2}\right\} .
\end{gathered}
$$

This expression is deduced from the action (1) by replacing the microscopic field $\mathbf{R}$ by the macroscopic one $\mathbf{r}$, followed by the usual rescaling of the field: $\mathbf{r} \mapsto$ $Z_{k}^{1 / 2} \kappa^{-1 / 2} \mathbf{r}$. We have introduced two field renormalization "constants": $Z_{k}$ and $Z_{k}^{\tau}=Z_{k} \rho \kappa^{-1}$ and defined two elastic running coupling constants $\lambda_{k}=Z_{k}^{2} \tilde{\lambda} \kappa^{-2}$ and $\mu_{k}=Z_{k}^{2} \tilde{\mu} \kappa^{-2}$ as well as a running extension parameter $\zeta_{k}=\kappa^{1 / 2} Z_{k}^{-1 / 2} \tilde{\zeta}$ that, together, parametrize the RG flow. The ansatz (24) corresponds to the next-to-leading order [64] of the derivative expansion of the effective action whose next orders would enclose higher powers of time and space derivatives of the field $\mathbf{r}$. Our choice to limit ourselves to the form (24) is justified by the fact that we give here priority to the description of infra-red behaviour of the flat phase, where only low-order derivative terms are expected to play a significant role, leaving the investigation of the short distance behaviour to further work [52]. The ability of our ansatz (24) to describe the former regime has received justifications in recent works. For instance Braghin and Hasselmann [41, 43] have studied the flat phase of classical membranes by means of a NPRG approach taking into account the full momentum dependence of the interaction vertices of Eq.(24) thus involving infinite order derivative terms. They have shown that the values of physical quantities, as the critical exponent $\eta$ characterizing the momentum dependence of the bending rigidity in this phase, were not impacted by derivative terms beyond the order considered here. Note, moreover, that we have also performed an expansion of the effective action in powers of the order parameter fields $\partial_{\gamma} \mathbf{r}$ and $\partial_{\tau} \mathbf{r}$ at the lowest non-trivial order. A complete treatment of the next-to-leading order of the derivative expansion considered here would require to use for the "kinetic " part of the action not just field renormalization constants $Z_{k}$ and $Z_{k}^{\tau}$ but full functions $Z_{k}\left(\partial_{\tau} \mathbf{r}, \partial_{\gamma} \mathbf{r}\right)$ and $Z_{k}^{\tau}\left(\partial_{\tau} \mathbf{r}, \partial_{\gamma} \mathbf{r}\right)$ and for the elastic - potential - part of the action a full "elasticity" function $U_{k}\left(\partial_{\gamma} \mathbf{r}\right)$. Beyond its simplicity, the justification of our choice comes from the fact that, at least in the classical case, the critical exponent $\eta$ in the flat phase is strictly independent of powers of the field higher than four [44]; one expects this result to remain true in the quantum case. Being given the ansatz Eq.(24) its RG flow is given by (see [32] and references therein):

$$
\partial_{t} \Gamma_{k}[\mathbf{r}]=\frac{T}{2} \sum_{\omega_{n}} \int_{\mathbf{q}} \partial_{t} R_{k}(\tilde{\mathbf{q}})\left(\Gamma_{k}^{(2)}[\mathbf{r} ; \tilde{\mathbf{q}},-\tilde{\mathbf{q}}]+R_{k}(\tilde{\mathbf{q}})\right)_{i i}^{-1}
$$

where Eq.(20) has been adapted to the quantum case [65] In this expression $\tilde{\mathbf{q}}$ stands for $\tilde{\mathbf{q}}=\left(\mathbf{q}, \omega_{n}\right)$ and the trace in Eq.(20) has been written explicitly as an integral over momentum $\mathbf{q}$, a sum over the frequencies $\omega_{n}=2 \pi n / \beta$ as well as an implicit sum over the field indices $i$.

\section{E. The cut-off function $R_{k}$}

It remains to specify the functional dependence of the cut-off function $R_{k}(\tilde{\mathbf{q}})$. We consider the following expression:

$$
R_{k}(\tilde{\mathbf{q}})=Z_{k}\left(q^{4}+\frac{\omega_{n}^{2}}{\Delta_{k}^{2}}\right) \mathcal{R}\left(\frac{q^{4}+\omega_{n}^{2} / \Delta_{k}^{2}}{k^{4}}\right)
$$

where $\mathcal{R}($.$) is a function such that R_{k}\left(\mathbf{q}, \omega_{n}=0\right)$ fulfills the conditions Eq.(13a)-(13b). In Eq.(26) $\Delta_{k}^{2}=Z_{k} / Z_{k}^{\tau}$ that equals $\Delta_{\Lambda}^{2}=\kappa / \rho$ at the lattice scale. The function $R_{k}(\tilde{\mathbf{q}})$ acts now as a infra-red cut-off for both lowmomenta and low-frequencies. With this form its respects the dispersion relation of the flexural modes that dominate the flat phase. As a consequence modes with momenta $q^{2}<k^{2}$ and/or frequencies $\left|\omega_{n}\right|<\Delta_{k} k^{2}$ are eliminated while those with momenta $q^{2}>k^{2}$ and/or frequencies $\left|\omega_{n}\right|>\Delta_{k} k^{2}$ are left untouched.

As for the question of the choice of the function $\mathcal{R}$ entering in Eq.(26) it is generally a complex one. Indeed due to the vanishing property of the cut-off function $R_{k}(\mathbf{q})$ when $k \rightarrow 0$, the physical quantities extracted from Eq.(20) in this limit are a priori cut-off independent. This statement is however only true when the effective action $\Gamma_{k}$ is treated exactly. Yet, as said above, in practice one has to resort to approximations of the effective action that destroy the cut-off independence of 
$\Gamma_{k=0}=\Gamma$ as computed from Eq.(20), and thus that of any physical quantity. This situation has lead to the search for the best or optimal regulator that would provide the weakest cut-off dependence (see for instance [34] for a review). This question of the optimization of the cut-off is a delicate one since one should precise what should be optimized: stability [46, 53-55], cut-off independence of the RG flow [34], speed of convergence of the field and field/derivative expansion, accuracy of physical quantities [56], etc. At the lowest order of the derivative expansion, called Local Potential Approximation, where the field renormalizations are neglected, these different criterions coincide and have lead to pick out the $\Theta$ cutoff Eq.(15) as the optimal one. However beyond order $\partial^{2}$ the $\Theta$ cut-off no longer regulates the RG flow and no equivalent optimal cut-off has been proposed yet (see however $[57,58]$ for optimized cut-offs that both regulate the flow and encompass several cut-offs previously considered). In the present work, since previous computations performed on classical membranes have shown an extremely weak dependence of the results with respect to the field/field derivative content of the action [40, 41, 43] we opt for the usual $\Theta$ cut-off that displays both formal simplicity and easy computability.

Note finally, in Eq.(26), the presence of the field renormalization $Z_{k}$ in front of the cut-off function that ensures that $Z_{k}$ explicitly disappears from the RG equations where it only appears through its derivative $\eta_{k}=$ $-\left(1 / Z_{k}\right) \partial_{t} Z_{k}$ - see below. At a fixed point $\eta_{k}$ reaches a fixed value $\eta$. This provides the scaling of $Z_{k}: Z_{k} \sim k^{-\eta}$. In the same way $Z_{k}^{\tau}$ scales at a fixed point as $Z_{k}^{\tau} \sim k^{-\tilde{\eta}}$.

\section{F. Power counting}

Action (24) involves the terms that are supposed to play the most important role at long distances; this allows power counting considerations at a putative fixed point. In terms of running momentum scale $k$ one has $\left[\partial_{\gamma}\right]=k$ and $\left[\partial_{\tau}\right]=\left[\partial_{\gamma}\right]^{z}=k^{z}$ were we have introduced the dynamical critical exponent $z$. Using the fixed point relations $Z_{k} \sim k^{-\eta}$ and $Z_{k}^{\tau} \sim k^{-\tilde{\eta}}$ one gets the field dimension $[\mathbf{r}]=D-4+\eta+z$ or indifferently $[\mathbf{r}]=D+\tilde{\eta}-z$ from which one deduces the relation: $\tilde{\eta}=\eta+2(z-2)$. As for the elastic coupling constants one has: $[\lambda]=[\mu]=-(D-4+z+2 \eta)$ and for the (squared) extension factor $\left[\zeta^{2}\right]=D-2+\eta+z$. Simple dimensional analysis - i.e. $\eta=\tilde{\eta}=0$ - leads to $z=2$ and $[\lambda]=[\mu]=-(D-2)$. This defines $D=2$ as the upper critical dimension of the theory (24) as it will be confirmed by our RG analysis.

\section{THE RG FLOW}

\section{A. General principle}

We now derive the RG equations for the different coupling constants entering in Eq.(24). In order to do this one has to define these coupling constants as functional derivatives of $\Gamma_{k}$ taken in a specific field configuration. We choose a flat ground state configuration, given by Eq.(2), since it corresponds to that with a vanishing external source, the situation that one wants to describe precisely. In Fourier space it reads:

$$
r_{k, j}^{f}\left(\mathbf{q}, \omega_{n}\right)=-i \zeta_{k} \delta_{n, 0} \delta_{\gamma, j} \frac{\partial}{\partial q_{\gamma}} \delta(\mathbf{q})
$$

where we recall that $\gamma=1 \ldots D$ and $j=1 \ldots d$. Note that the configuration $\mathbf{r}_{k}^{f}$ also runs with $k$ due to the running of the extension parameter $\zeta_{k}$.

With the action Eq.(24) a generic coupling constant $g_{k, i j}$ can be defined as some coefficient in the expansion of $\Gamma_{k, i j}^{(2)}[\mathbf{r} ; \tilde{\mathbf{p}},-\tilde{\mathbf{p}}]$, with $\tilde{\mathbf{p}}=\left(\mathbf{p}, \omega_{m}\right)$, in powers of $p$ and $\omega_{m}$ (the explicit definition of each coupling constant are given in Appendix(A 1), Eqs.(A1a)-(A1d)). Explicitly one has:

$$
g_{k, i j}=\lim _{\tilde{\mathbf{p}} \rightarrow \mathbf{0}} \frac{1}{a ! b !} \frac{d^{a}}{d\left(\omega_{m}^{2}\right)^{a}} \frac{d^{b}}{d\left(p^{2}\right)^{b}}\left\{\left.\Gamma_{k, i j}^{(2)}[\mathbf{r} ; \tilde{\mathbf{p}},-\tilde{\mathbf{p}}]\right|_{\mathbf{r}_{k}^{f}}\right\} .
$$

The flow of the coupling constant $g_{k, i j}$ is then deduced by taking a derivative with respect to $t$ :

$$
\begin{aligned}
\partial_{t} g_{k, i j}= & \lim _{\tilde{\mathbf{p}} \rightarrow \mathbf{0}} \frac{1}{a ! b !} \frac{d^{a}}{d\left(\omega_{m}^{2}\right)^{a}} \frac{d^{b}}{d\left(p^{2}\right)^{b}}\left\{\left.\partial_{t} \Gamma_{k, i j}^{(2)}[\mathbf{r} ; \tilde{\mathbf{p}},-\tilde{\mathbf{p}}]\right|_{\mathbf{r}_{k}^{f}}\right. \\
& \left.+\left.\int_{\tilde{\mathbf{q}}} \Gamma_{k, i j l}^{(3)}[\mathbf{r} ; \tilde{\mathbf{p}},-\tilde{\mathbf{p}}, \tilde{\mathbf{q}}] \partial_{t} r_{k}^{l}(\tilde{\mathbf{q}})\right|_{\mathbf{r}_{k}^{f}}\right\}
\end{aligned}
$$

It results from the previous equation that it is sufficient to know the flow of $\Gamma_{k, i j}^{(2)}[\mathbf{r} ; \tilde{\mathbf{p}},-\tilde{\mathbf{p}}]$ which is easily obtained by taking the second derivative of Eq.(25) with respect to $r_{i}(\tilde{\mathbf{p}})$ and $r_{j}(\tilde{\mathbf{p}})$, what leads to:

$$
\begin{aligned}
\partial_{t} \Gamma_{k, i j}^{(2)}[\mathbf{r} ; \tilde{\mathbf{p}},-\tilde{\mathbf{p}}]= & -\frac{1}{2} \widehat{\partial}_{t}\left\{\int_{\tilde{\mathbf{q}}} G_{k, a b}[\mathbf{r}, \tilde{\mathbf{q}}] \Gamma_{k, i j a b}^{(4)}[\mathbf{r} ; \tilde{\mathbf{p}},-\tilde{\mathbf{p}}, \tilde{\mathbf{q}},-\tilde{\mathbf{q}}]\right. \\
& \left.-\int_{\tilde{\mathbf{q}}} G_{k, a b}[\mathbf{r}, \tilde{\mathbf{q}}] \Gamma_{k, i a c}^{(3)}[\mathbf{r} ; \tilde{\mathbf{p}},-\tilde{\mathbf{q}}, \tilde{\mathbf{q}}-\tilde{\mathbf{p}}] G_{k, c d}[\mathbf{r}, \tilde{\mathbf{q}}-\tilde{\mathbf{p}}] \Gamma_{k, j b d}^{(3)}[\mathbf{r} ;-\tilde{\mathbf{p}}, \tilde{\mathbf{q}}, \tilde{\mathbf{p}}-\tilde{\mathbf{q}}]\right\}
\end{aligned}
$$

where

$$
G_{k, a b}[\mathbf{r}, \tilde{\mathbf{q}}]=\left[\Gamma_{k, a b}^{(2)}[\mathbf{r} ; \tilde{\mathbf{q}},-\tilde{\mathbf{q}}]+R_{k, a b}(\tilde{\mathbf{q}})\right]^{-1} .
$$

In Eq.(29) and (30) the vertices $\Gamma_{k, b j d}^{(3)}$ and $\Gamma_{k, a b i j}^{(4)}$ are 
further functional derivatives of $\Gamma_{k}$. Their expressions in the flat configuration $\mathbf{r}_{k, f}$, Eq.(27), are given in Appendix A 3, Eqs.(A8a) and (A8b).

\section{B. RG equations}

For the search of fixed points it is convenient to write the flow equations in terms of dimensionless coupling constants. We thus define $\zeta_{k}^{2}=Z_{k}^{-1} k^{D-2+z} \bar{\zeta}_{k}^{2}, \lambda_{k}=$ $Z_{k}^{2} k^{-(D-4+z)} \bar{\lambda}_{k}$ and $\mu_{k}=Z_{k}^{2} k^{-(D-4+z)} \bar{\mu}_{k}$. Their flows are given by:

$$
\begin{aligned}
& \partial_{t} \bar{\zeta}_{k}^{2}=-\left(D-2+z+\eta_{k}\right) \bar{\zeta}_{k}^{2}+\frac{4 A_{D}}{D} \times \\
& \quad\left\{(D-1) \frac{4 \bar{\mu}_{k}+\bar{\lambda}_{k} D}{2 \bar{\mu}_{k}+\bar{\lambda}_{k} D} \bar{l}_{010}^{D+2}+\right. \\
&\left.\quad \frac{6 \bar{\mu}_{k}+(D+2) \bar{\lambda}_{k}}{2 \bar{\mu}_{k}+\bar{\lambda}_{k} D} \bar{l}_{001}^{D+2}+(d-D) \bar{l}_{100}^{D+2}\right\} \\
& \partial_{t} \bar{\mu}_{k}=\left(D-4+2 \eta_{k}+z\right) \bar{\mu}_{k}+\frac{2 A_{D}}{D(D+2)} \times \\
&\left\{4\left(3 \bar{\mu}_{k}+\bar{\lambda}_{k}\right)^{2} \bar{l}_{002}^{D+4}+4 D \bar{\mu}_{k}\left(2 \bar{\mu}_{k}+\bar{\lambda}_{k}\right) \bar{l}_{011}^{D+4}+\right. \\
&\left.2 \bar{\mu}_{k}^{2}\left(D^{2}+2 D-8\right) \bar{l}_{020}^{D+4}+4 \bar{\mu}_{k}^{2}(d-D) \bar{l}_{200}^{D+4}\right\} \\
& \partial_{t} \bar{\lambda}_{k}=\left(D-4+z+2 \eta_{k}\right) \bar{\lambda}_{k}+\frac{2 A_{D}}{D(D+2)} \times \\
&\left\{-8 \bar{\mu}_{k}\left(2 \bar{\mu}_{k}+\bar{\lambda}_{k}\right) \bar{l}_{011}^{D+4}+\right. \\
& \quad(d-D)\left[4 \bar{\mu}_{k}^{2}+4(D+2) \bar{\mu}_{k} \bar{\lambda}_{k}+D(D+2) \bar{\lambda}_{k}^{2}\right] \bar{l}_{200}^{D+4}+ \\
& {\left[4(3 D+2) \bar{\mu}_{k}^{2}+\left(D^{2}+D-2\right)\left(8 \bar{\mu}_{k} \bar{\lambda}_{k}+D \bar{\lambda}_{k}^{2}\right)\right] \bar{l}_{020}^{D+4}+} \\
& {\left.\left[36 \bar{\mu}_{k}^{2}+12(D+4) \bar{\mu}_{k} \bar{\lambda}_{k}+\left(D^{2}+6 D+12\right) \bar{\lambda}_{k}^{2}\right] \bar{l}_{002}^{D+4}\right\} }
\end{aligned}
$$

where $A_{D}^{-1}=2^{D+1} \pi^{D / 2} \Gamma(D / 2)$ and $\Gamma(\ldots)$ is the Euler's gamma function. The equation for $\eta_{k}$ is too long to be given here while $\tilde{\eta}_{k}$ is discussed below. The set of equations (32a)-(32c), together with $\eta_{k}$ and $\tilde{\eta}_{k}$, generalize to the quantum case those derived in [40] in the classical case.

\section{Threshold functions}

In Eqs.(32a)-(32c) $\bar{l}_{a b c}^{D}$ stands for the dimensionless counterpart of the so-called dimensionful "threshold functions" $l_{a b c}^{D}-$ see Appendix B:

$$
l_{a b c}^{D}=-\frac{T}{4 A_{D}} \widehat{\partial}_{t} \sum_{\omega_{n}} \int_{\mathbf{q}} \frac{1}{\left[P_{0}(\tilde{\mathbf{q}})\right]^{a}\left[P_{1}(\tilde{\mathbf{q}})\right]^{b}\left[P_{2}(\tilde{\mathbf{q}})\right]^{c}}
$$

where $P_{i}(\tilde{\mathbf{q}})=P(\tilde{\mathbf{q}})+m_{i k}^{2} q^{2}, i=0,1,2$ and $P(\tilde{\mathbf{q}})=$ $Z_{k} q^{4}+Z_{k}^{\tau} \omega_{n}^{2}+R_{k}(\tilde{\mathbf{q}})$. The squared masses $m_{i k}^{2}, i=1,2$ given by $m_{1 k}^{2}=\mu_{k} \zeta_{k}^{2}$ and $m_{2 k}^{2}=\left(2 \mu_{k}+\lambda_{k}\right) \zeta_{k}^{2}$ are associated with the transverse and longitudinal phonon modes while the squared mass $m_{0 k}^{2} \equiv 0$ is associated with the flexural mode. The threshold functions are the fundamental ingredient of the effective average action formalism. First, they control the integration over fluctuations of lower and lower momenta when $k$ is decreased. Second, they encompass the nonperturbative content of the RG flow since they include the propagators that are nonpolynomial functions of the masses and thus of the coupling constants. Third, they govern the phenomenon of $d e-$ coupling of massive modes. This refers to a situation in which coexist particles - or excitations - with well separated masses, e.g., a large one $M$ and a smaller one $m$ the latter being possibly equal to zero. It states that when the theory is probed at a momentum scale $p \ll M$ the Green functions of the full theory are identical, up to inverse powers of $M^{2}$, to those computed from an effective theory where the heavy mass has been cancelled out. Within the effective average action framework this phenomenon occurs along the RG flow when the running momentum scale $k$ gets lower than the large running mass $M_{k}$. Indeed in this case, the threshold functions decrease typically as inverse powers of $M_{k}$, see below. Roughly this relies on the fact that $\partial_{k} R_{k}(\tilde{\mathbf{q}})$ acts as an ultraviolet cut-off that restricts the values of $q$ contributing to the momenta integrals to the range $[0, k]$; in particular the high momentum lattice scale $\Lambda$ disappears from the flow. This elects the running scale $k$ as the only reference scale to which $M_{k}$ can be compared, what leads to the announced result.

\section{The temporal anomalous dimension $\tilde{\eta}$}

We discuss here the RG flow of the field renormalization $Z_{k}^{\tau}$ and derive the expression of the running "temporal" anomalous dimension $\tilde{\eta}_{k}$ defined by: $\tilde{\eta}_{k}=$ $-1 / Z_{k}^{\tau} \partial_{t} Z_{k}^{\tau}$. The quantity $Z_{k}^{\tau}$ is defined as the coefficient of $\omega_{m}^{2}$ in the expansion of $\Gamma_{k}^{(2)}$ in powers of the external momentum $p$ and frequency $\omega_{m}$, at vanishing $\tilde{\mathbf{p}}$ :

$$
Z_{k}^{\tau}=\lim _{\tilde{\mathbf{p}} \rightarrow 0} \frac{d}{d \omega_{m}^{2}}\left[\left.\Gamma_{k, D+1, D+1}^{(2)}[\mathbf{r}, \tilde{\mathbf{p}},-\tilde{\mathbf{p}}]\right|_{\mathbf{r}_{k}^{f}}\right] .
$$

As a consequence of the discussion of subsection IV A its flow is essentially given by the $\omega_{m}^{2}$-derivative of $\partial_{t} \Gamma_{k, i j}^{(2)}[\mathbf{r} ; \tilde{\mathbf{p}},-\tilde{\mathbf{p}}]$, see Eq.(30), taken in the configuration $\mathbf{r}_{k}^{f}$, Eq.(27). Since, at this order, the vertices are frequency independent - see Appendix (A 3), Eqs.(A8a)(A8b) - the only contribution to the renormalization of $Z_{k}^{\tau}$ can come from the term $G \Gamma^{(3)} G \Gamma^{(3)}$ in the right-hand size of Eq.(30), involving $G_{k, c d}[\mathbf{r}, \tilde{\mathbf{q}}-\tilde{\mathbf{p}}]$ that carries the external frequency $\omega_{m}$. However the vertex functions $\Gamma_{k, b j d}^{(3)}$ carry at least one power of the external momentum p so that the putative contribution to the renormalization of $Z_{k}^{\tau}$ vanishes in the limit $\tilde{\mathbf{p}} \rightarrow \mathbf{0}$ and one has:

$$
\tilde{\eta}_{k}=0 \text {. }
$$




\section{E. The RG flow in the flat phase}

We now derive the RG equations in the flat phase. They can be obtained by following explicitly the RG flow Eqs.(32a)-(32c) in the infra-red, i.e. $k \rightarrow 0$, regime with initial conditions taken in this phase. This shows that the extension parameter $\zeta_{k}$ reaches a finite value while its dimensionless counterpart $\bar{\zeta}_{k}$ diverges. On the other hand, the coupling constants $\lambda_{k}$ and $\mu_{k}$ vanish while $\bar{\lambda}_{k}$ and $\bar{\mu}_{k}$ reach fixed point values. As a consequence the dimensionless masses $\bar{m}_{1 k}^{2}=\bar{\mu}_{k} \bar{\zeta}_{k}^{2}$ and $\bar{m}_{2 k}^{2}=\left(2 \bar{\mu}_{k}+\bar{\lambda}_{k}\right) \bar{\zeta}_{k}^{2}$ get large in the infra-red. We thus meet the conditions of the decoupling phenomenon where the phonon modes get out the RG flow leaving the flexural mode to govern it. This provides a direct way to get the RG equations in the flat phase, i.e. by considering the limit: $\bar{m}_{1 k}$, $\bar{m}_{2 k} \rightarrow \infty$. As this can be explicitly checked in the case of the $\Theta$ cut-off - see Eqs.(B9a) and (B9b) in Appendix (B 2) - the "massive" dimensionless threshold functions $\bar{l}_{0 b c}^{D}$ (and $\bar{n}_{0 b c}^{D}$ - see below) decay as powers of $\bar{m}_{1 k}^{2}$ and $\bar{m}_{2 k}^{2}$, what reflects, on the dimensionless threshold functions, the decoupling phenomenon. In this limit one gets simplified RG equations where only the part of the flow associated with the flexural mode appears, what implies the presence of threshold functions $\bar{l}_{a 00}^{D}$ (and $\bar{n}_{a 00}^{D}$, see below) alone. The RG flow for $\zeta_{k}^{2}$ is given by:

$$
\partial_{t} \bar{\zeta}_{k}^{2}=-\left(D-2+z+\eta_{k}\right) \bar{\zeta}_{k}^{2}+\frac{4 A_{D}}{D}(d-D) \bar{l}_{100}^{D+2} .
$$

This equation controls the different phases, and phase transitions, occurring in the phase diagram of membranes. It admits a non-trivial unstable fixed point $\bar{\zeta}_{*}^{2}$ that corresponds to the crumpling-to-flat transition by which we are not interested in here. For $\bar{\zeta}_{k}^{2}>\bar{\zeta}_{*}^{2}$ the flow drives $\bar{\zeta}_{k}^{2}$ toward its infinite value limit that defines the flat phase. The other RG equations in this phase are given by:

$\partial_{t} \bar{\mu}_{k}=\left(D-4+2 \eta_{k}+z\right) \bar{\mu}_{k}+\frac{8 A_{D}}{D(D+2)} \bar{\mu}_{k}^{2}(d-D) \bar{l}_{200}^{D+4}$

$$
\begin{aligned}
\partial_{t} \bar{\lambda}_{k}= & \left(D-4+z+2 \eta_{k}\right) \bar{\lambda}_{k}+\frac{2 A_{D}}{D(D+2)}(d-D) \times \\
& \left(4 \bar{\mu}_{k}^{2}+4(D+2) \bar{\mu}_{k} \bar{\lambda}_{k}+D(D+2) \bar{\lambda}_{k}^{2}\right) \bar{l}_{200}^{D+4} .
\end{aligned}
$$

As for the anomalous dimension $\eta_{k}$ it is given by :

$$
\begin{gathered}
\eta_{k}=\frac{2 A_{D}}{D(D+2)\left(2 \bar{\mu}_{k}+\bar{\lambda}_{k}\right)}\left\{2(D+2)\left(2 \bar{\mu}_{k}+\bar{\lambda}_{k}\right)^{2} \bar{n}_{200}^{D}+\right. \\
\left.\left(4\left(D^{2}-2 D-2\right) \bar{\mu}_{k}\left(\bar{\mu}_{k}+\bar{\lambda}_{k}\right)-D(D+2) \bar{\lambda}_{k}^{2}\right) \bar{l}_{100}^{D}\right\} .
\end{gathered}
$$

In this last equation we have introduced the threshold functions $\bar{n}_{a b c}^{D}$ which are the dimensionless counterparts of the dimensionful threshold functions $n_{a b c}^{D}$ - see Appendix B:

$$
n_{a b c}^{D}=-\frac{T}{4 A_{D}} \widehat{\partial}_{t} \sum_{\omega_{n}} \int_{\mathbf{q}} \frac{q^{2} \partial_{q^{2}} P(\tilde{\mathbf{q}})}{\left[P_{0}(\tilde{\mathbf{q}})\right]^{a}\left[P_{1}(\tilde{\mathbf{q}})\right]^{b}\left[P_{2}(\tilde{\mathbf{q}})\right]^{c}} .
$$

\section{F. Quantum to classical crossover}

The RG equations Eqs.(37a)-(37b) and (38) account for the effects of both classical and quantum fluctuations when the running scale $k$ varies. In order to quantify the importance of quantum fluctuations, it is convenient to define a dimensionless effective, running, "temperature" $\bar{T}_{k}$ through:

$$
T=\Delta_{k} k^{2} \bar{T}_{k}
$$

such that $\bar{\beta}_{k}=1 / \bar{T}_{k}$ appears as the dimensionless thickness of the time integral in Eq.(24) which characterizes the importance of quantum fluctuations. The definition of $\bar{T}_{k}$ leads to consider a typical momentum - the thermal momentum scale $-k_{T}$ via the relation $\bar{T}_{k_{T}} \sim 1$ that, using Eq.(40) and $\Delta_{k_{T}} \sim \sqrt{\kappa / \rho}$ yields:

$$
k_{T} \sim \sqrt{\frac{k_{B} T}{\hbar} \sqrt{\frac{\rho}{\kappa}}} .
$$

For effective "temperatures" $\bar{T}_{k} \ll 1-$ or equivalently for momenta $k \gg k_{T}$ - the dimensionless thickness $\bar{\beta}_{k}$ of the time integral extends from 0 to $\infty$ and quantum effects are important. Conversely for temperatures $\bar{T}_{k} \gg 1-$ or equivalently for momenta $k \ll k_{T}$ - this thickness $\bar{\beta}_{k}$ vanishes and fluctuations are fully classical. More precisely, let's consider the action $S[\mathbf{R}]$, Eq.(1), expressed in terms of dimensionless quantities:

$$
\begin{aligned}
S & {[\overline{\mathbf{R}}]=\int_{0}^{\bar{\beta}_{k}} d \bar{\tau} \int d^{D} \bar{x}\left\{\frac{\bar{\rho}}{2}\left(\partial_{\bar{\tau}} \overline{\mathbf{R}}\right)^{2}+\frac{\bar{\kappa}}{2}\left(\partial_{\bar{\gamma}} \partial_{\bar{\gamma}} \overline{\mathbf{R}}\right)^{2}\right.} \\
& \left.+\frac{\overline{\tilde{\mu}}}{4}\left(\partial_{\bar{\gamma}} \overline{\mathbf{R}} \cdot \partial_{\bar{\nu}} \overline{\mathbf{R}}-\overline{\tilde{\zeta}}^{2} \delta_{\gamma, \nu}\right)^{2}+\frac{\overline{\tilde{\lambda}}}{8}\left(\partial_{\bar{\gamma}} \overline{\mathbf{R}} \cdot \partial_{\bar{\gamma}} \overline{\mathbf{R}}-D \overline{\tilde{\zeta}}^{2}\right)^{2}\right\} .
\end{aligned}
$$

When $\bar{T}_{k} \gg 1$ or, equivalently, when $\bar{\beta}_{k}$ vanishes the only configurations of $\mathbf{R}(\overline{\mathbf{x}}, \bar{\tau})$ that contribute to the functional integral Eq.(19) are those that minimize locally the kinetic term $\left(\partial_{\bar{\tau}} \overline{\mathbf{R}}\right)^{2}$, i.e. those that are timeindependent. As a consequence the effective average action $\Gamma_{k}[\overline{\mathbf{r}}]$ resulting from this functional integral is also time-independent. The $\bar{\beta}_{k}$ term then factors out and the effective action takes the form:

$$
\begin{aligned}
\Gamma_{k}[\overline{\mathbf{r}}] & =\bar{\beta}_{k} \int d^{D} \bar{x}\left\{\frac{Z_{k}}{2}\left(\partial_{\bar{\gamma}} \partial_{\bar{\gamma}} \overline{\mathbf{r}}\right)^{2}\right. \\
& \left.+\frac{\bar{\mu}_{k}}{4}\left(\partial_{\bar{\gamma}} \overline{\mathbf{r}} \cdot \partial_{\bar{\nu}} \overline{\mathbf{r}}-\bar{\zeta}_{k}^{2} \delta_{\gamma, \nu}\right)^{2}+\frac{\bar{\lambda}_{k}}{8}\left(\partial_{\bar{\gamma}} \overline{\mathbf{r}} \cdot \partial_{\bar{\gamma}} \overline{\mathbf{r}}-D \bar{\zeta}_{k}^{2}\right)^{2}\right\} .
\end{aligned}
$$


Redefining $\overline{\mathbf{r}} \rightarrow \bar{\beta}_{k}^{-1 / 2} \overline{\mathbf{r}}$ and introducing the "classical" coupling constants $\bar{\lambda}_{k}^{c l}=\bar{\beta}_{k}^{-1} \lambda_{k}, \bar{\mu}_{k}^{c l}=\bar{\beta}_{k}^{-1} \mu_{k}$ and $\bar{\zeta}_{k}^{c l}=\bar{\beta}_{k}^{1 / 2} \zeta_{k}$, one gets the effective action of classical membranes:

$$
\begin{aligned}
\Gamma_{k}[\overline{\mathbf{r}}] & =\int d^{D} \bar{x}\left\{\frac{Z_{k}}{2}\left(\partial_{\bar{\gamma}} \partial_{\bar{\gamma}} \overline{\mathbf{r}}\right)^{2}\right. \\
& \left.+\frac{\bar{\mu}_{k}^{c l}}{4}\left(\partial_{\bar{\gamma}} \overline{\mathbf{r}} \cdot \partial_{\bar{\nu}} \overline{\mathbf{r}}-\bar{\zeta}_{k}^{c l} \delta_{\gamma, \nu}^{2}\right)^{2}+\frac{\bar{\lambda}_{k}^{c l}}{8}\left(\partial_{\bar{\gamma}} \overline{\mathbf{r}} \cdot \partial_{\bar{\gamma}} \overline{\mathbf{r}}-D \bar{\zeta}_{k}^{c l^{2}}\right)^{2}\right\} .
\end{aligned}
$$

This illustrates the well-know phenomenon of thermal dimensional reduction from a $(D+1)$-dimensional to a $D$ dimensional model associated with the quantum to classical crossover that occurs when the temperature increases [59].

It is instructive to interpret this phenomenon from the RG point of view. As said in section (IIIE), we have chosen a cut-off that acts both on momenta and frequencies. Using the $\Theta$ cut-off consists in taking, in Eq.(26), $\mathcal{R}(Y)=\left(Y^{-1}-1\right) \Theta(1-Y)$ which leads to:

$R_{k}(\tilde{\mathbf{q}})=Z_{k}\left(k^{4}-\left(q^{4}+\frac{\omega_{n}^{2}}{\Delta_{k}^{2}}\right)\right) \Theta\left(1-\left(\frac{q^{4}}{k^{4}}+\frac{\omega_{n}^{2}}{\Delta_{k}^{2} k^{4}}\right)\right)$.

With this form of cut-off it is easy to verify that the term $\partial_{t} R_{k}(\tilde{\mathbf{q}})$ entering in the threshold functions $l_{a b c}^{D}$ and $n_{a b c}^{D}$ enforces strictly [66] that only the range of momenta/frequencies $0 \leq q^{4} / k^{4}+\omega_{n}^{2} /\left(\Delta_{k}^{2} k^{4}\right) \leq 1$ contributes to the RG flow. Once the integral is performed on momentum $q$ the constraint on $\omega_{n}$ reads: $\omega_{n} /\left(\Delta_{k} k^{2}\right) \leq 1$. Using $\omega_{n}=2 \pi n T$ and the relation Eq. (40) this inequality implies that the frequency modes that effectively contribute to the RG flow are those characterized by integers $n$ obeying: $n \leq n_{\max }=\left\lfloor 1 /\left(2 \pi \bar{T}_{k}\right)\right\rfloor$ where $\lfloor\ldots\rfloor$ denotes the floor function. Finally the RG flow of $\bar{T}_{k}$ is easily obtained from Eq.(40) and the flow of $\Delta_{k}$ :

$$
\partial_{t} \bar{T}_{k}=-z \bar{T}_{k}
$$

which means that $\bar{T}_{k}$ is, as expected, a relevant variable [67]. Thus, as $k$ decreases, the high-frequency modes are progressively integrated out and disappear from the $\mathrm{RG}$ flow. This can be seen, again, as a kind of decoupling of the non-vanishing (or "massive") Matsubara frequencies at low momenta leading to a situation dominated by the classical $-\omega_{n}=0-$ mode.

\section{G. The RG flow at vanishing temperature}

We now discuss the RG equations in the flat phase. We start by considering the limit of vanishing effective temperatures $\bar{T}_{k} \ll 1$ or large running momentum scale $k \gg k_{T}$. In this case all frequency modes contribute. Technically the sums over frequencies in the dimensionless threshold functions $\bar{l}_{a b c}^{D}$ and $\bar{n}_{a b c}^{D}$, see Appendix B 1,
Eqs.(B9a) and (B9b), are replaced by integrals over continuous frequencies:

$$
\bar{T}_{k} \sum_{\bar{\omega}_{n}} \rightarrow \int_{-\infty}^{\infty} \frac{d \bar{\omega}}{2 \pi}
$$

Replacing, in the RG flow equations, Eqs.(37a)-(37b) and (38), the threshold functions by their simplified expressions, Eqs.(B11a)-(B11b), and $z$ by its value $z=$ $2-\eta_{k} / 2$ one obtains the equations for any dimension $D$. For simplicity we concentrate here on the physical $D=2$ case in which one has:

$$
\begin{aligned}
\partial_{t} \bar{\mu}_{k} & =\frac{3}{2} \eta_{k} \bar{\mu}_{k}+\frac{1}{32 \pi}(d-2) \bar{\mu}_{k}^{2}\left(1-\frac{\eta_{k}}{8}\right) \\
\partial_{t} \bar{\lambda}_{k} & =\frac{3}{2} \eta_{k} \bar{\lambda}_{k} \\
& +\frac{1}{32 \pi}(d-2)\left(2 \bar{\lambda}_{k}^{2}+4 \bar{\lambda}_{k} \bar{\mu}_{k}+\bar{\mu}_{k}^{2}\right)\left(1-\frac{\eta_{k}}{8}\right) \\
\eta_{k} & =\frac{48 \bar{\mu}_{k}\left(\bar{\lambda}_{k}+\bar{\mu}_{k}\right)}{9 \bar{\mu}_{k}\left(\bar{\lambda}_{k}+\bar{\mu}_{k}\right)+128 \pi\left(\bar{\lambda}_{k}+2 \bar{\mu}_{k}\right)} .
\end{aligned}
$$

These equations are the nonperturbative generalization of those found previously perturbatively [12, 13, 28].

\section{Large $d$ expansion}

As argued in the general discussion of section (III C) our equations should reproduce the leading order in a $1 / d$ expansion. In this case one assumes that the coupling constants behave as $1 / d$. At leading order in $1 / d$ Eqs.(48a)-(48c) then simplify into, after the rescaling $\left(\bar{\mu}_{k}, \bar{\lambda}_{k}\right) \rightarrow\left(\bar{\mu}_{k} / d, \bar{\lambda}_{k} / d\right):$

$$
\begin{aligned}
\partial_{t} \bar{\mu}_{k} & =\frac{1}{32 \pi} \bar{\mu}_{k}^{2} \\
\partial_{t} \bar{\lambda}_{k} & =\frac{1}{32 \pi}\left(2 \bar{\lambda}_{k}^{2}+4 \bar{\lambda}_{k} \bar{\mu}_{k}+\bar{\mu}_{k}^{2}\right) \\
\eta_{k} & =\frac{3}{8 \pi d} \frac{\bar{\mu}_{k}\left(\bar{\lambda}_{k}+\bar{\mu}_{k}\right)}{\left(\bar{\lambda}_{k}+2 \bar{\mu}_{k}\right)}
\end{aligned}
$$

It is convenient to write the RG equations (49a)-(49b) in terms of the Young modulus $\overline{\mathcal{Y}}_{k}=4 \bar{\mu}_{k}\left(\bar{\mu}_{k}+\bar{\lambda}_{k}\right) /\left(2 \bar{\mu}_{k}+\right.$ $\left.\bar{\lambda}_{k}\right)$. One obtains:

$$
\partial_{t} \overline{\mathcal{Y}}_{k}=\frac{3}{128 \pi} \overline{\mathcal{Y}}_{k}^{2}
$$

which, restoring the coupling constants $\kappa$ and $\rho$ coincides with the expression obtained by Guinea et al.[13] and up to a factor 2 already noted in [13] - with the equation derived by San Jose et al.[12]. Note that $\eta_{k}$ provides the 
flow of the bending rigidity modulus $\kappa$ in the original action Eq.(1) through the relation $\eta_{k}=-\kappa^{-1} \partial_{t} \kappa$. One gets [68]:

$$
\partial_{t} \kappa=-\frac{3}{32 \pi} \overline{\mathcal{Y}}_{k} \kappa
$$

which, restoring the coupling constants $\kappa$ and $\rho$, coincides again, up to a factor 2 , with the equation obtained in [12].

\section{Weak-coupling expansion}

The equations Eqs.(48a)-(48c) can also be expanded in powers of the coupling constants $\bar{\lambda}_{k}$ and $\bar{\mu}_{k}$. One finds, at leading non-trivial order:

$$
\begin{aligned}
\partial_{t} \bar{\mu}_{k} & =\frac{3}{2} \eta_{k} \bar{\mu}_{k}+\frac{1}{32 \pi}(d-2) \bar{\mu}_{k}^{2} \\
\partial_{t} \bar{\lambda}_{k} & =\frac{3}{2} \eta_{k} \bar{\lambda}_{k}+\frac{1}{32 \pi}(d-2)\left(2 \bar{\lambda}_{k}^{2}+4 \bar{\lambda}_{k} \bar{\mu}_{k}+\bar{\mu}_{k}^{2}\right) \\
\eta_{k} & =\frac{3}{8 \pi} \frac{\bar{\mu}_{k}\left(\bar{\mu}_{k}+\bar{\lambda}_{k}\right)}{\bar{\lambda}_{k}+2 \bar{\mu}_{k}} .
\end{aligned}
$$

In the particular case where $d=3$, and restoring the coupling constants $\kappa$ and $\rho$, these equations coincide with those derived by Kats and Lebedev in [28]. There is, however, a drastic difference that concerns the sign of $\eta_{k}$ that we find to be reversed with respect to theirs. This leads to a major difference as for the behaviour of membranes at long distances - see below.

\section{3. $R G$ flow in $D=2$ and $d=3$}

We have studied the RG equations Eqs.(48a)-(48c), i.e. searched for the fixed points and analyzed the structure of the flow, in the physical $D=2$ and $d=3$ case. They display one physical fixed point which corresponds to the gaussian one with $\bar{\mu}=\bar{\lambda}=0$. This fixed point is attractive in the infra-red, see Fig.(1), in agreement with the fact that $D=2$ is, according to previous considerations, supposed to be the upper critical dimension of the theory. This contradicts the result obtained in [28] that the gaussian fixed point is repulsive.

Now since, in the infra-red, the RG flow is essentially controlled by the gaussian fixed point we can restrict our study to the vicinity of this fixed point and consider the perturbative limit Eqs.(52a)-(52c) of our equations.

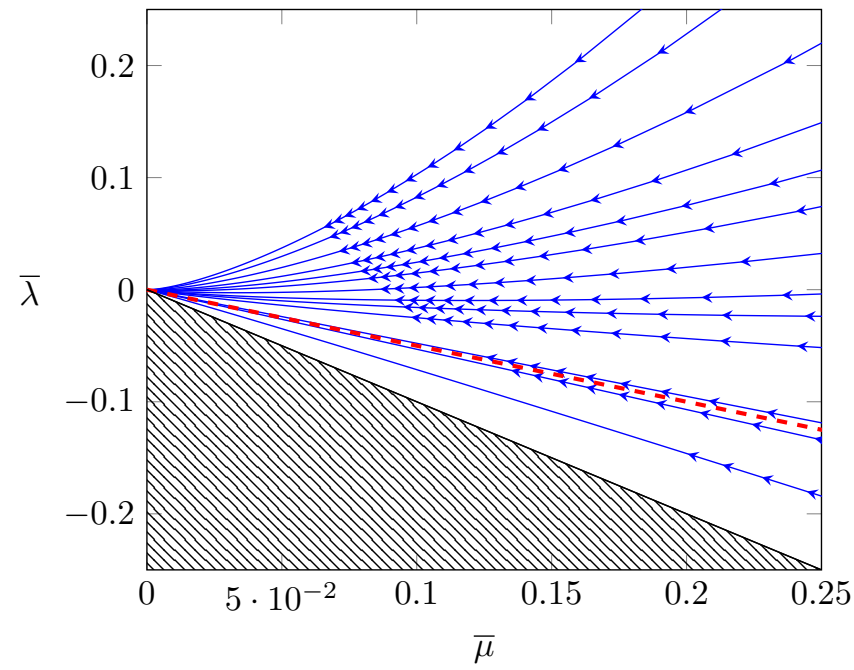

FIG. 1: The RG flow in the $(\bar{\lambda}, \bar{\mu})$ plane. The shaded area corresponds to the forbidden region $\bar{\lambda}+\bar{\mu}<0$; the bordeline $\bar{\lambda}=-\bar{\mu}$ is repulsive as the line $\bar{\mu}=0$. The dashed line corresponds to the attractive line $\bar{\lambda}=-\bar{\mu} / 2$ along which all trajectories converge.

They read in $d=3$ :

$$
\begin{aligned}
\partial_{t} \bar{\mu}_{k} & =\frac{\bar{\mu}_{k}^{2}\left(19 \bar{\lambda}_{k}+20 \bar{\mu}_{k}\right)}{32 \pi\left(\bar{\lambda}_{k}+2 \bar{\mu}_{k}\right)} \\
\partial_{t} \bar{\lambda}_{k} & =\frac{2 \bar{\lambda}_{k}^{3}+26 \bar{\lambda}_{k}^{2} \bar{\mu}_{k}+27 \bar{\lambda}_{k} \bar{\mu}_{k}^{2}+2 \bar{\mu}_{k}^{3}}{32 \pi\left(\bar{\lambda}_{k}+2 \bar{\mu}_{k}\right)} \\
\eta_{k} & =\frac{3}{8 \pi} \frac{\bar{\mu}_{k}\left(\bar{\mu}_{k}+\bar{\lambda}_{k}\right)}{\bar{\lambda}_{k}+2 \bar{\mu}_{k}} .
\end{aligned}
$$

It is instructive to compute the flow of the ratio $\bar{\mu}_{k} / \bar{\lambda}_{k}$ which given by:

$$
\partial_{t}\left(\frac{\bar{\mu}_{k}}{\bar{\lambda}_{k}}\right)=-\frac{\bar{\mu}_{k}}{32 \pi \bar{\lambda}_{k}^{2}}\left(\bar{\lambda}_{k}+\bar{\mu}_{k}\right)\left(2 \bar{\lambda}_{k}+\bar{\mu}_{k}\right) .
$$

It is then easy to shows that the RG flow admits one attractive line, $\bar{\lambda}=-\bar{\mu} / 2$, see Fig.(1), and two repulsive ones, $\bar{\lambda}=-\bar{\mu}$ and $\bar{\mu}=0$. The behaviour of the flow in the vicinity of the gaussian fixed point along the attractive line is given by:

$$
\begin{gathered}
\partial_{t} \bar{\mu}_{k}=\frac{7}{32 \pi} \bar{\mu}_{k}^{2} \\
\eta_{k}=\frac{\bar{\mu}_{k}}{8 \pi} .
\end{gathered}
$$

The effective coupling constant $\bar{\mu}_{k}$ decreases at long distances, again in agreement with the fact that $D=2$ is expected to be the upper critical dimension of quantum membranes and in disagreement with [28] where an 
increasing effective coupling constant is found at long distances. Integrating then the flow equations Eqs.(55a)(55b) provides the expressions of the momentum dependent bending rigidity $\kappa(q) \sim|\ln q|^{4 / 7}$, in agreement with [14], and the momentum dependent coupling $\bar{\mu}(q) \sim$ $|\ln q|^{-1}$. These logarithmic behaviours, together with the considerations of section (IIB) about the fluctuations in the flat phase, imply that polymerized membranes are not destabilized by quantum fluctuations at $T=0$. Note finally that, as for the momentum dependent anomalous dimension $\eta(q)$, we also predicts a vanishing - logarithmic - behaviour $\eta(q) \sim|\ln q|^{-1}$ that contrasts with the prediction of [24] that leads to a finite value $-\eta=2-$ at vanishing temperature.

Again one can rewrite Eqs.(53a) and (53b) in terms of the Young modulus:

$$
\partial_{t} \overline{\mathcal{Y}}_{k}=\frac{21}{128 \pi} \overline{\mathcal{Y}}_{k}^{2}
$$

that integrates into:

$$
\overline{\mathcal{Y}}_{k}=\frac{\overline{\mathcal{Y}}_{\Lambda}}{1-\frac{21 t}{128 \pi} \overline{\mathcal{Y}}_{\Lambda}} .
$$

The Young modulus is generally used to define a quantum Ginzburg momentum scale $k_{G}^{q}$ that separates a strong from a weak coupling regime. Defining this scale as the one at which the Young modulus looses half its initial value:

$$
\frac{|\delta \overline{\mathcal{Y}}|}{\overline{\mathcal{Y}}_{\Lambda}}=\frac{\left|\overline{\mathcal{Y}}_{k}-\overline{\mathcal{Y}}_{\Lambda}\right|}{\overline{\mathcal{Y}}_{\Lambda}} \simeq \frac{1}{2}
$$

one obtains:

$$
k_{G}^{q} \sim \Lambda e^{-\frac{64 \pi}{21 \overline{\mathcal{Y}}_{\Lambda}}} .
$$

Our expression is close to that obtained in the large $d$ approach of [13] with a change of coefficient in the argument of the exponential [69] due to the non-vanishing anomalous dimension within our computation.

\section{H. The RG flow at high temperatures}

\section{General equations}

We now consider the limit of high effective temperatures $\bar{T}_{k} \gg 1-$ or low momentum scales $k \ll k_{T}$. As said previously, in this case, due to the thermal decoupling of high frequency modes, only the vanishing frequency mode contributes to the RG flow. Technically the sums in the threshold functions $\bar{l}_{a b c}^{D}$ and $\bar{n}_{a b c}^{D}$, see Eqs.(B10a) and $(\mathrm{B} 10 \mathrm{~b})$, reduce to the value $\omega_{n}=0$. In this case the threshold functions are given by Eqs.(B12a)-(B12b). Using these quantities and the set of classical coupling constants : $\bar{\lambda}_{k}^{c l}=\bar{\beta}_{k}^{-1} \bar{\lambda}_{k}, \bar{\mu}_{k}^{c l}=\bar{\beta}_{k}^{-1} \bar{\mu}_{k}$ and $\overline{\mathcal{Y}}_{k}^{c l}=\bar{\beta}_{k}^{-1} \overline{\mathcal{Y}}_{k}$ one obtains from Eqs.(37a)-(37b) and (38):

$$
\begin{gathered}
\partial_{t} \bar{\mu}_{k}^{c l}=\left(D-4+2 \eta_{k}\right) \bar{\mu}_{k}^{c l}+4(d-D) \bar{\mu}_{k}^{c l}{ }^{2} \widetilde{A}_{D} \\
\partial_{k} \bar{\lambda}_{k}^{c l}=\left(D-4+2 \eta_{k}\right) \bar{\lambda}_{k}^{c l}+(d-D) \times \\
\left(4 \bar{\mu}_{k}^{c l}+4(D+2) \bar{\mu}_{k}^{c l} \bar{\lambda}_{k}^{c l}+D(D+2) \bar{\lambda}_{k}^{c l}\right) \widetilde{A}_{D} \\
\eta_{k}=\frac{16(D+4)\left(D^{2}-1\right) \overline{\mathcal{Y}}_{k}^{c l} A_{D}}{D^{2}\left(D^{2}+6 D+8\right)+16\left(D^{2}-1\right) \overline{\mathcal{Y}}_{k}^{c l} A_{D}}
\end{gathered}
$$

with $\widetilde{A}_{D}=16 A_{D}\left(8+D-\eta_{k}\right) / D(D+2)(D+4)(D+8)$.

These equations coincide exactly - up to redefinitions of the coupling constants - with those derived in [40] for classical membranes. They have been shown to agree with those obtained from a perturbative expansion performed around $D=4[19,21]$ as well as with those obtained within a large $d$ approach [21, 22, 60]. Thus our formalism smoothly interpolates between the full quantum situation and the full classical one.

\section{2. $R G$ flow in $D=2$ and $d=3$}

In physical dimensions the RG flow is given by:

$$
\begin{gathered}
\partial_{t} \bar{\mu}_{k}^{c l}=2\left(\eta_{k}-1\right) \bar{\mu}_{k}^{c l}+\frac{\bar{\mu}_{k}^{c l}}{60 \pi}\left(10-\eta_{k}\right) \\
\partial_{k} \bar{\lambda}_{k}^{c l}=2\left(\eta_{k}-1\right) \bar{\lambda}_{k}^{c l}+ \\
\frac{1}{60 \pi}\left(2 \bar{\lambda}_{k}^{c l}{ }^{2}+4 \bar{\mu}_{k}^{c l} \bar{\lambda}_{k}^{c l}+\bar{\mu}_{k}^{c l}{ }^{2}\right)\left(10-\eta_{k}\right) \\
\eta_{k}=\frac{6 \bar{\mu}_{k}^{c l}\left(\bar{\lambda}_{k}^{c l}+\bar{\mu}_{k}^{c l}\right)}{\bar{\mu}_{k}^{c l}\left(\bar{\lambda}_{k}^{c l}+\bar{\mu}_{k}^{c l}\right)+4 \pi\left(\bar{\lambda}_{k}^{c l}+2 \bar{\mu}_{k}^{c l}\right)} .
\end{gathered}
$$

These equations have been solved in [40]. They admit a stable fixed point with coordinates $\bar{\mu}_{*}^{c l} \simeq 6.21, \bar{\lambda}_{*}^{c l} \simeq$ -3.10 with the associated critical exponent $\eta^{*} \simeq 0.849$.

The flow of the ratio $\bar{\mu}_{k}^{c l} / \bar{\lambda}_{k}^{c l}$ is given by:

$$
\begin{aligned}
\partial_{t}\left(\frac{\bar{\mu}_{k}^{c l}}{\bar{\lambda}_{k}^{c l}}\right)= & -\frac{\bar{\mu}_{k}^{c l}\left(\bar{\lambda}_{k}^{c l}+\bar{\mu}_{k}^{c l}\right)\left(2 \bar{\lambda}_{k}^{c l}+\bar{\mu}_{k}^{c l}\right)}{15 \pi \bar{\lambda}_{k}^{c l}{ }^{2}} \times \\
& \frac{\bar{\mu}_{k}^{c l}\left(\bar{\lambda}_{k}^{c l}+\bar{\mu}_{k}^{c l}\right)+10 \pi\left(\bar{\lambda}_{k}^{c l}+2 \bar{\mu}_{k}^{c l}\right)}{\bar{\mu}_{k}^{c l}\left(\bar{\lambda}_{k}^{c l}+\bar{\mu}_{k}^{c l}\right)+4 \pi\left(\bar{\lambda}_{k}^{c l}+2 \bar{\mu}_{k}^{c l}\right)}
\end{aligned}
$$

One deduces that the RG flow admits, as in the vanishing temperature case, one attractive line, $\bar{\lambda}=-\bar{\mu} / 2$, and two repulsive ones $\bar{\lambda}=-\bar{\mu}$ and $\bar{\mu}=0$ that are identical to those found in this last case. 
Here, again, one can exhibit a characteristic - classical - Ginzburg momentum scale separating a strong from a weak coupling regime. We consider the flow of the dimensionful Young modulus [70] at dominant order in $\mathcal{Y}_{k}^{c l}$. It is given by:

$$
\partial_{t} \mathcal{Y}_{k}^{c l}=\frac{7}{8 \pi} \frac{\mathcal{Y}_{k}^{c l^{2}}}{k^{2}}
$$

that integrates into:

$$
\mathcal{Y}_{k}^{c l}=\frac{\mathcal{Y}_{\Lambda}^{c l}}{1-\frac{7}{8 \pi} \mathcal{Y}_{\Lambda}^{c l}\left(\frac{1}{2 \Lambda^{2}}-\frac{1}{2 k^{2}}\right)} .
$$

From Eq.(58), and restoring the physical units, we get:

$$
k_{G}^{c l}=\sqrt{\frac{7 \tilde{\mathcal{Y}}_{\Lambda}^{c l}}{8 \pi} \frac{k_{B} T}{\kappa^{2}}} .
$$

\section{APPLICATION TO GRAPHENE}

We now illustrate the different crossovers encountered in previous sections in the context of the physics of free standing graphene.

\section{A. Initial conditions}

We first specify the initial conditions of the RG flow. We take as microscopical characteristics of graphene (see, e.g., [16] and references therein) : $\kappa \simeq 1 \mathrm{eV}, \rho \simeq 7.6 \times$ $10^{-7} \mathrm{~kg} . \mathrm{m}^{-2}, \mu \simeq 3 \lambda \simeq 9 \mathrm{eV} . \AA^{-2}$, the lattice parameter $a \simeq 1.4 \AA$ and $\mathcal{Y} \simeq 20.6 \mathrm{eV} . \AA^{-2}$. They are related to the dimensionless bare quantum coupling constants at the lattice scale $\Lambda=a^{-1}: \bar{\lambda}_{\Lambda}=\hbar \lambda /\left(\rho^{1 / 2} \kappa^{3 / 2}\right)$ and $\bar{\mu}_{\Lambda}=$ $\hbar \mu /\left(\rho^{1 / 2} \kappa^{3 / 2}\right)$. As the classical bare coupling constants they are defined by $\bar{\lambda}_{\Lambda}^{c l}=\bar{T}_{\Lambda} \bar{\lambda}_{\Lambda}$ and $\bar{\mu}_{\Lambda}^{c l}=\bar{T}_{\Lambda} \bar{\mu}_{\Lambda}$. The bare dimensionless temperature $\bar{T}_{\Lambda}$ is related to $\Delta_{\Lambda}=$ $\sqrt{\kappa / \rho}$ by Eq.(40). This leads to:

$$
\bar{T}_{\Lambda}=\sqrt{\frac{\rho}{\kappa}} \frac{k_{B} T}{\hbar} a^{2} .
$$

\section{B. Crossover momenta}

Once the temperature $T$ has been chosen the crossover scales are completely determined. One takes $T=10 \mathrm{~K}$ since the temperature below which quantum fluctuations are expected to be dominant is estimated to be around 70 - $90 \mathrm{~K}$ [16]. For an initial temperature of $10 \mathrm{~K}$ the thermal momentum scale $k_{T}=a^{-1} e^{-t_{T}}$ is given by :

$$
k_{T}=\sqrt{\frac{k_{B} T}{\hbar} \sqrt{\frac{\rho}{\kappa}}} \sim a^{-1} e^{-1.44} \sim 0.17 \AA^{-1}
$$

that corresponds to a length of a few lattice spacing.

As for the quantum Ginzburg momentum scale it is given by:

$$
k_{G}^{q}=\Lambda e^{-\frac{64 \pi \kappa^{\frac{3}{2}} \sqrt{\rho}}{21 \mathcal{Y} \hbar}} \sim a^{-1} e^{-15.4} \ll 1
$$

which is very small with respect to $k_{T}$, in agreement with [13] but in contradiction with [24] that predicts $k_{G}^{q} \sim$ $0.1 \AA^{-1}$. The origin of this contradiction lies on the fact that $D=2$ being, within our computation, the upper critical dimension of quantum membranes, $k_{G}^{q}$ takes the usual - exponentially decreasing - form of a mass-gap in such a dimension, see Eq.(59). This is not the case in [24].

Since $k_{G}^{q} \ll k_{T}$, i.e. the weak/strong coupling quantum crossover occurs far in the region where thermal fluctuations already dominate, it cannot be observed and has no role in the physical behaviour of the graphene sheet.

In the classical regime the Ginzburg momentum scale is given by:

$$
k_{G}^{c l}=\sqrt{\frac{7 \tilde{\mathcal{Y}}_{\Lambda}^{c l}}{8 \pi} \frac{k_{B} T}{\kappa^{2}}} \simeq a^{-1} e^{-2.30} \sim 0.07 \AA^{-1} .
$$

which implies that $k_{G}^{c l}<k_{T}$.

\section{Crossover behaviour of coupling constants}

The behaviour of the system under the RG flow is as follows: starting in a quantum regime governed by a gaussian fixed point, it goes through the quantum to classical crossover. It then enters a classical weak-coupling regime controlled by a gaussian fixed point and is finally driven toward a strong coupling infra-red classical regime governed by a non-trivial fixed point.

These behaviours are first illustrated on the RG flow of the coupling constants $\lambda$ and $\mu$. In Fig.(2) we have displayed the flow of the quantum coupling constants $\bar{\lambda}_{k}$ and $\bar{\mu}_{k}$ as functions of the RG "time" $-t=\ln \Lambda / k$. Starting at finite values they first decrease slowly toward their (vanishing) gaussian fixed point values, their behaviour being essentially controlled by the (anomalous) dimensional part of the vanishing temperature RG flow, see Eqs.(48a)-(48c). This vanishing behaviour is then amplified when the quantum to classical RG time $t_{T}$ is reached since, there, the flow starts to be controlled by the finite temperature RG equations Eqs.(37a)-(37b) and (38) and, in particular, by non-trivial part of the flow proportional to the effective temperature $\bar{T}_{k}$ carried by the threshold functions, see Eqs.(B12a)-(B12b).

In Fig.(3) we have displayed the flow of the classical dimensionless coupling constants $\bar{\mu}_{k}^{c l}$ and $\bar{\lambda}_{k}^{c l}$ as functions of the RG"time" $-t=\ln \Lambda / k$. These coupling constants undergo - roughly - a symmetrical behaviour. From their very definition including thermal factor $\bar{\beta}_{k}^{-1}$, they start 


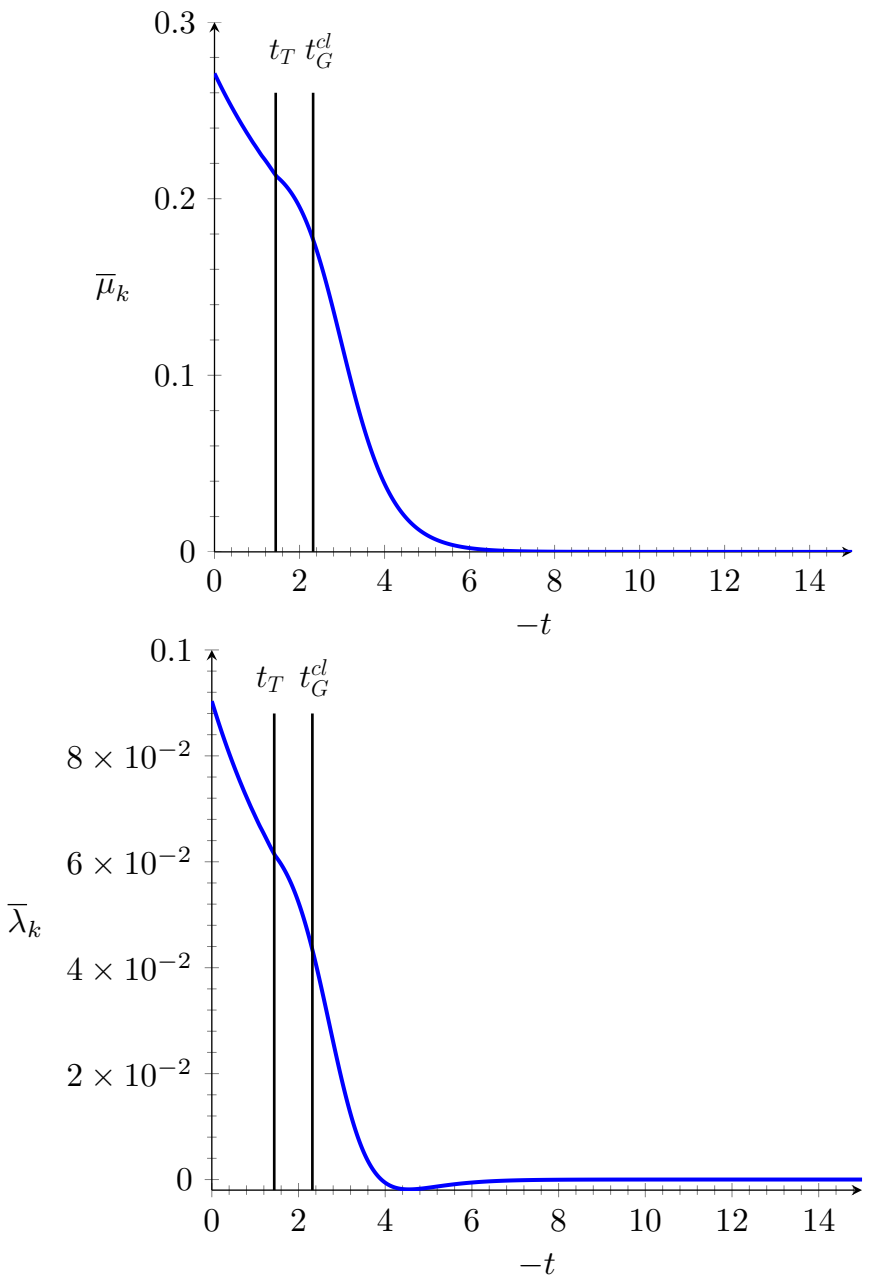

FIG. 2: Evolution of the dimensionless quantum coupling constants $\bar{\mu}_{k}$ and $\bar{\lambda}_{k}$ with $\mathrm{RG}$ time $-t=\ln \Lambda / k$. The left vertical line corresponds to the quantum to classical crossover momentum scale $k_{T}$ that is located at $-t_{T}=1.44$; the right vertical line corresponds to the classical Ginzburg momentum scale $k_{G}^{c l}$ that is located at $-t_{G}^{c l}=2.30 . t_{T}$ marks a crossover between a regime controlled by the quantum gaussian fixed point and a regime where the vanishing behaviour is enhanced by the effective temperature $\bar{T}_{k}$.

from almost vanishing values and first increase slowly with the effective temperature $\bar{T}_{k}$. Then, when the quantum to classical RG time $t_{T}$ is reached, their behaviour begins to be controlled by the classical RG Eqs.(61a)$(61 \mathrm{~b})$ and $(61 \mathrm{c})$ which amplifies this tendency. At $t_{G}^{c l}$, the coupling constants enter a regime where the nonlinearities of the flow start to play a role. Finally the coupling constants reach their asymptotic values $\bar{\lambda}_{*}^{c l}$ and $\bar{\mu}_{*}^{c l}$ associated with the classical fixed point.

\section{Crossover behaviour of physical quantities}

The previous crossovers can also be observed for different relevant physical quantities: the anomalous
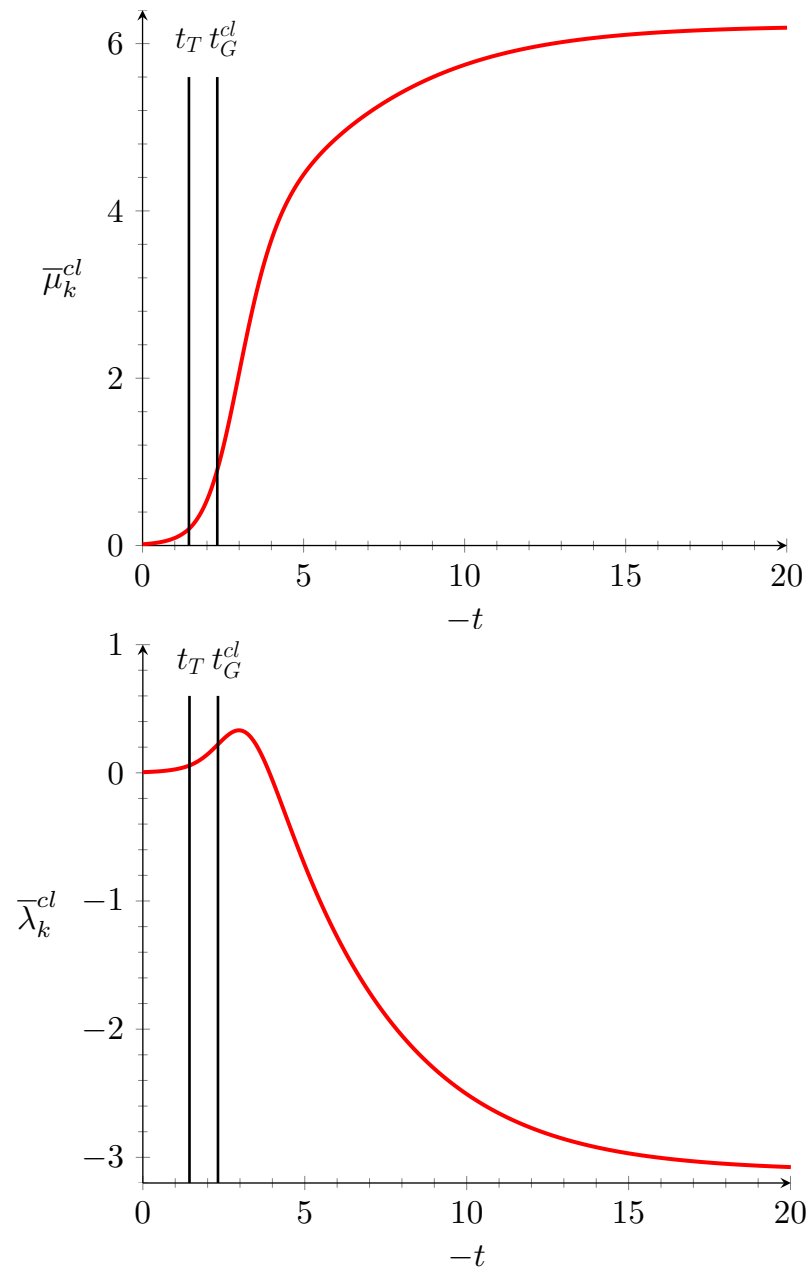

FIG. 3: Evolution of the dimensionless classical coupling constants $\bar{\mu}_{k}^{c l}$ and $\bar{\lambda}_{k}^{c l}$ with $\mathrm{RG}$ time $-t=\ln \Lambda / k$. $t_{G}^{c l}$ marks a clear crossover between a regime controlled by a classical gaussian fixed point $\left(\bar{\mu}_{*}^{c l}=\bar{\lambda}_{*}^{c l}=0\right)$ and a regime controlled by the infra-red non-trivial classical fixed point $\left(\bar{\mu}_{*}^{c l} \simeq 6.21\right.$, $\left.\bar{\lambda}_{*}^{c l} \simeq-3.10\right)$.

dimension $\eta_{k}$, Fig.(4), the dimensionless classical bulk modulus $\bar{K}_{k}^{c l}=\bar{\lambda}_{k}^{c l}+\bar{\mu}_{k}^{c l}$, Fig.(5), and the dimensionless classical Young modulus $\overline{\mathcal{Y}}_{k}^{c l}=4 \bar{\mu}_{k}^{c l}\left(\bar{\mu}_{k}^{c l}+\bar{\lambda}_{k}^{c l}\right) /\left(2 \bar{\mu}_{k}^{c l}+\bar{\lambda}_{k}^{c l}\right)$, Fig.(6). They all share the same kind evolution: relaxation toward vanishing values in the quantum regime (not apparent on $K$ and $\mathcal{Y}$ due to the smallness of the initial conditions), transition toward a classical regime and stabilization in the infra-red, classical, behaviour. Note the oscillations of $\eta_{k}$ at early RG time $t$ on Fig.(4) due to the use of the discontinuous $\Theta$ cut-off.

Finally we have represented the Poisson ratio $\nu_{k}=$ $\lambda_{k} /\left(\lambda_{k}+2 \mu_{k}\right)$, Fig.(7). Its evolution is different from other quantities since, due to its expression and the "universal" attractive line $\lambda=-\mu / 2$, it does converge toward the value $\nu_{k}=-1 / 3$ in all regimes of temperatures. The main effect of the crossovers is to change its rate of con- 


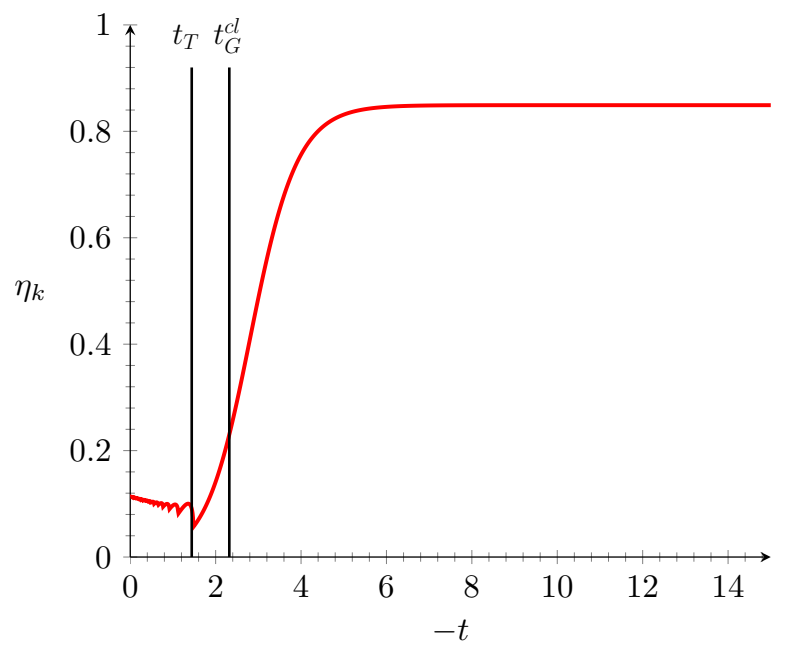

FIG. 4: Evolution of the running anomalous dimension $\eta_{k}$ with the $\mathrm{RG}$ time $-t=\ln \Lambda / k . \quad \eta_{k}$ evolves from a quasigaussian (quantum) regime to a non-trivial (classical) regime where it reaches the fixed point value $\eta^{*}=0.849$. The oscillations at the beginning of the flow are an artifact related to the use of the $\Theta$ cut-off.

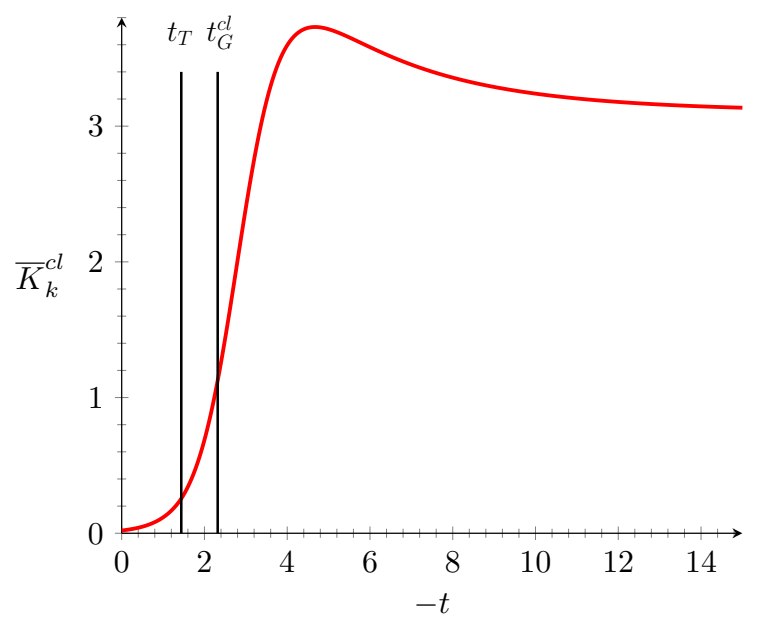

FIG. 5: Evolution of the dimensionless classical bulk modulus $\bar{K}_{k}^{c l}$ the with the RG time $-t=\ln \Lambda / k$ that converges to the value $\bar{K}_{*}^{c l} \simeq 3.11$.

vergence: $\nu_{k}$ evolves slowly in the quantum regime and more rapidly in the classical regime.

\section{CONCLUSION}

In this article we have investigated quantum polymerized membranes within a NPRG framework. We have first derived, from the RG equations describing generic fluctuating membranes, those relevant to study the flat phase. We have then analyzed the corresponding RG flow that smoothly interpolates between a quantum and a classical regime. We have shown that, at vanishing tem-

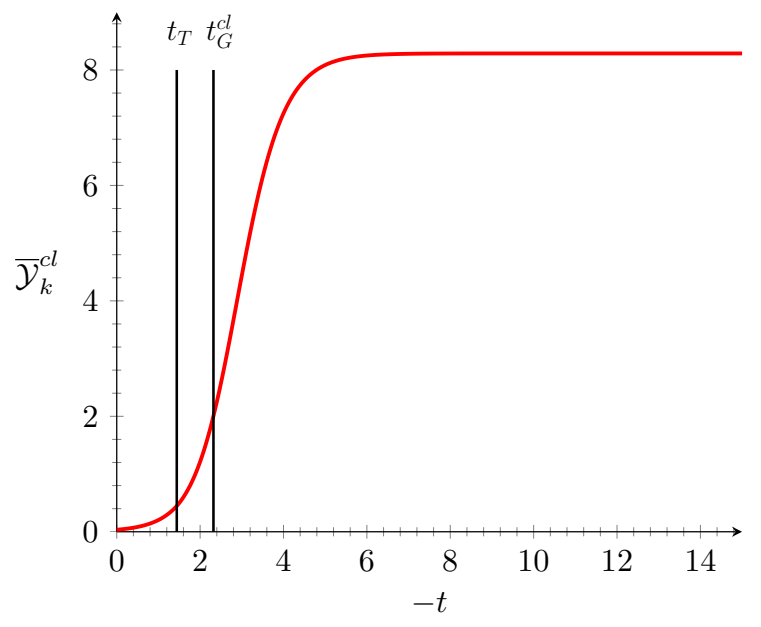

FIG. 6: Evolution of the dimensionless classical Young modulus $\overline{\mathcal{Y}}_{k}^{c l}$ with the RG time $-t=\ln \Lambda / k$ that converges to the value $\overline{\mathcal{Y}}_{*}^{c l} \simeq 8.30$.

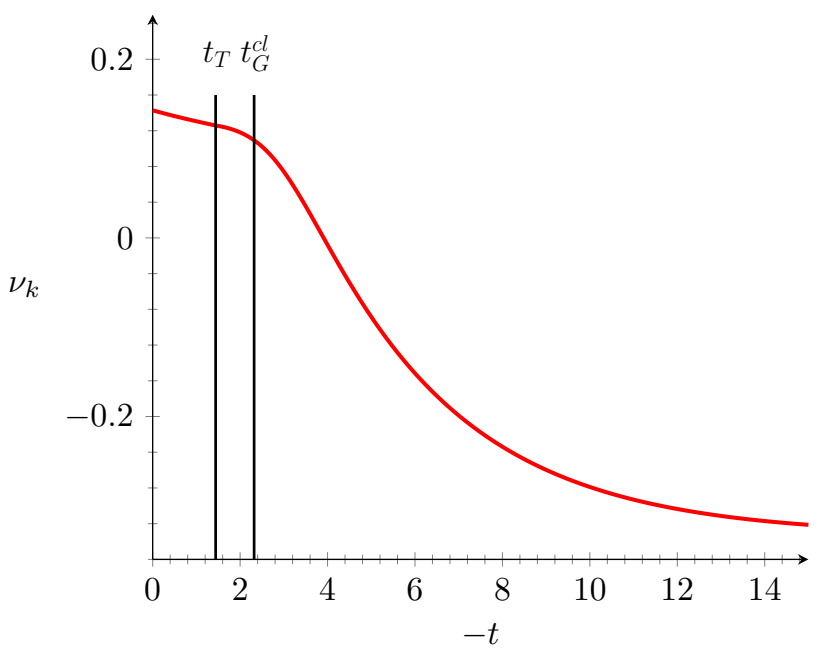

FIG. 7: Evolution of the Poisson ratio $\nu_{k}$ with the RG time $-t=\ln \Lambda / k$ that converges to the value $\nu_{*}=-1 / 3$.

peratures, the infra-red behaviour is governed by an attractive gaussian fixed point. We have then described the quantum to classical crossover leading to the usual nontrivial infra-red behaviour associated with stable classical membranes. In each, quantum or classical, regime, we have exhibited the typical momentum scale associated with the crossover between the weak and strong coupling regimes. Finally we have illustrated these different properties in the case of free standing graphene.

We have shown, in the context of the physics of membranes, the ability of the NPRG approach to continuously interpolate between a quantum regime governed by a gaussian fixed point and a classical regime governed by a strongly interacting fixed point. This relies on the nonperturbative character of the formalism used. As emphasized previously approximations are nevertheless necessary and their nature must be carefully adjusted in accor- 
dance with the physical context. The present approach has been performed at the next-to-leading order in the derivative expansion together with a field-expansion. Although somewhat preliminary it has allowed us to recover (and in some cases to contest) previous perturbative weak-coupling or large $d$ - results and to improve them by inclusion of a nonvanishing anomalous dimension and nonperturbative contributions.

Further and systematic improvements of the present approach could be implemented. They would consist, for instance, in gradually enriching the effective action $\Gamma_{k}$ used by increasing powers of fields and field-derivatives. A more qualitative improvement would be to consider the whole momentum dependence of the effective action, an approach which has been partially performed in the classical case [41, 43] but for which a full treatment, in both the quantum and classical cases, remains a challenge. Such an approach would be particularly suitable to the computation of thermodynamical quantities, like the thermal expansion coefficient or the specific heat - as done for instance in [24] - since they precisely involve the full momentum content of the two-point correlation function. It would also allow to treat the effects of electronphonon interaction that involves a non-trivial momentum structure (see, e.g., [13]).

From a methodological point of view this kind of approach could also allow to clarify the relation between the NPRG approach used in this article and the SCBA approach performed in [24] that lead to contradicting results. We nevertheless think that this disagreement can hardly - but not impossibly - be solved by considering higher powers of field or field-derivatives as our result relies on the rather strong evidence of the existence of a stable gaussian fixed point at vanishing temperature. In our opinion this disagreement suggests to reconsider the arguments developed within the recent controversy involving the authors of [24, 30] and those of [28, 29] that precisely concerns the way the anomalous dimension is computed in [24]. More generally and finally an accurate control of the SCBA-SCSA and the NPRG approach in the context of quantum membranes - that appear, in several other contexts, notably that of disordered systems (see for instance [61]) as very complementary is highly desirable.

\section{Acknowledgments}

We thank B. Delamotte, P. Le Doussal, N. Dupuis and J.-P. Kownacki for helpful discussions and B. Amorim who provided us very valuable precisions about the technique used in [24].

\section{Appendix A: Coupling constants definitions and} vertex functions

\section{Coupling constants definitions}

The coupling constants are defined as functional derivatives of the effective action, considered in the configuration Eq.(27):

$$
\begin{aligned}
& \mu_{k}=\lim _{\tilde{\mathbf{p}} \rightarrow 0} \frac{1}{\zeta_{k}^{2}} \frac{d}{d p^{2}}\left[\left.\Gamma_{k, D-1, D-1}^{(2)}[\mathbf{r}, \tilde{\mathbf{p}},-\tilde{\mathbf{p}}]\right|_{\mathbf{r}_{k, f}}\right] \\
& \lambda_{k}=\lim _{\tilde{\mathbf{p}} \rightarrow 0}\left\{\frac{1}{\zeta_{k}^{2}} \frac{d}{d\left(p_{D-1}\right)^{2}}\left[\left.\Gamma_{k, D-1, D-1}^{(2)}[\mathbf{r}, \tilde{\mathbf{p}},-\tilde{\mathbf{p}}]\right|_{\mathbf{r}_{k, f}}\right]-\frac{1}{\zeta_{k}^{2}} \frac{d}{d p^{2}}\left[\left.\Gamma_{k, D-1, D-1}^{(2)}[\mathbf{r}, \tilde{\mathbf{p}},-\tilde{\mathbf{p}}]\right|_{\mathbf{r}_{k, f}}\right]\right\} \\
& Z_{k}=\lim _{\tilde{\mathbf{p}} \rightarrow 0} \frac{1}{2} \frac{d^{2}}{d\left(p^{2}\right)^{2}}\left[\left.\Gamma_{k, D+1, D+1}^{(2)}[\mathbf{r}, \tilde{\mathbf{p}},-\tilde{\mathbf{p}}]\right|_{\mathbf{r}_{k, f}}\right] \\
& Z_{k}^{\tau}=\lim _{\tilde{\mathbf{p}} \rightarrow 0} \frac{d}{d \omega_{m}^{2}}\left[\left.\Gamma_{k, D+1, D+1}^{(2)}[\mathbf{r}, \tilde{\mathbf{p}},-\tilde{\mathbf{p}}]\right|_{\mathbf{r}_{k, f}}\right] .
\end{aligned}
$$

The flow of $\zeta_{k}$ requires a specific treatment, see below.

\section{RG flow of $\zeta_{k}$}

Whereas the other coupling constants are defined from $\Gamma_{k}^{(2)}, \zeta_{k}$ is defined from the particular field configuration
Eq.(27). One of its characterization is that $\mathbf{r}_{k, f}$ is a minimum of the effective action:

$$
\left.\frac{\delta \Gamma_{k}[\mathbf{r}]}{\delta r_{j}(\tilde{\mathbf{q}})}\right|_{\mathbf{r}_{k, f}}=0 .
$$


However, because of translational invariance of the action density, any flat configuration of the form:

$$
r_{k, j}(\tilde{\mathbf{q}})=-i \sigma_{k} \delta_{n, 0} \delta_{\gamma, j} \frac{\partial}{\partial q_{\gamma}} \delta(\mathbf{q})
$$

where $\sigma_{k}$ represents any real number not necessarily equal to $\zeta_{k}$ satisfies the condition Eq.(A2). This last equation, therefore, does not properly define $\zeta_{k}$ as the extension parameter associated to the minimum of the effective action and cannot be used to compute its flow equation. To solve this problem, one has to remove the ambiguity associated to translational invariance. This can be done by replacing the condition Eq.(A2) by a condition where one performs the derivative of $\Gamma_{k}$ with respect to the tangent vectors to the membrane:

$$
\left.\frac{\delta \Gamma_{k}[\mathbf{r}]}{\delta \partial_{\alpha} r_{j}(\tilde{\mathbf{q}})}\right|_{\mathbf{r}_{k, f}}=0 .
$$

The flow of $\zeta_{k}$ is then deduced by taking a $t$-derivative of Eq.(A4), and a little algebra.

\section{Vertex functions}

The inverse propagator in the flat configuration Eq.(27) is given by:

$$
\begin{array}{r}
\Gamma_{k, i_{1}, i_{2}}^{(2)}\left[\mathbf{r}_{k, f} ; \tilde{\mathbf{q}}_{1}, \tilde{\mathbf{q}}_{2}\right]=\delta\left(\tilde{\mathbf{q}}_{1}+\tilde{\mathbf{q}}_{2}\right) \times\left(G_{F}^{-1}\left(\tilde{\mathbf{q}}_{1}\right) \mathcal{P}_{i_{1}, i_{2}}^{F}\left(\tilde{\mathbf{q}}_{1}\right)\right. \\
\left.+G_{\perp}^{-1}\left(\tilde{\mathbf{q}}_{1}\right) \mathcal{P}_{i_{1}, i_{2}}^{\perp}\left(\tilde{\mathbf{q}}_{1}\right)+G_{\|}^{-1}\left(\tilde{\mathbf{q}}_{1}\right) \mathcal{P}_{i_{1}, i_{2}}^{\|}\left(\tilde{\mathbf{q}}_{1}\right)\right)
\end{array}
$$

where we have defined the flexural, transversal and longitudinal projectors with respect to momentum $\mathbf{q}_{1}$ by:

$$
\left\{\begin{array}{l}
\mathcal{P}_{i_{1}, i_{2}}^{F}\left(\tilde{\mathbf{q}}_{1}\right)=\delta_{i_{1}, i_{2}}-\delta_{\alpha, i_{1}} \delta_{\alpha, i_{2}} \\
\mathcal{P}_{i_{1}, i_{2}}^{\perp}\left(\tilde{\mathbf{q}}_{1}\right)=\delta_{\alpha, i_{1}} \delta_{\alpha, i_{2}}-\delta_{\alpha, i_{1}} \delta_{\beta, i_{2}} \frac{q_{1, \alpha} q_{1, \beta}}{q_{1}^{2}} \\
\mathcal{P}_{i_{1}, i_{2}}^{\|}\left(\tilde{\mathbf{q}}_{1}\right)=\delta_{\alpha, i_{1}} \delta_{\beta, i_{2}} \frac{q_{1, \alpha} q_{1, \beta}}{q_{1}^{2}}
\end{array}\right.
$$

and where:

$$
\left\{\begin{array}{l}
G_{F}^{-1}\left(\tilde{\mathbf{q}}_{1}\right)=Z_{k} q_{1}^{4}+Z_{k}^{\tau} \omega_{1 n}^{2} \\
G_{\|}^{-1}\left(\tilde{\mathbf{q}}_{1}\right)=Z_{k} q_{1}^{4}+Z_{k}^{\tau} \omega_{1 n}^{2}+m_{2 k}^{2} q_{1}^{2} \\
G_{\perp}^{-1}\left(\tilde{\mathbf{q}}_{1}\right)=Z_{k} q_{1}^{4}+Z_{k}^{\tau} \omega_{1 n}^{2}+m_{1 k}^{2} q_{1}^{2}
\end{array}\right.
$$

with $m_{1 k}^{2}=\mu_{k} \zeta_{k}^{2}$ and $m_{2 k}^{2}=\left(2 \mu_{k}+\lambda_{k}\right) \zeta_{k}^{2}$.
As for the higher vertex functions in the flat configuration Eq.(27) there are given by:

$$
\begin{aligned}
& \Gamma_{k, i_{1}, i_{2}, i_{3}}^{(3)}\left[\mathbf{r}_{k, f} ; \tilde{\mathbf{q}}_{1}, \tilde{\mathbf{q}}_{2}, \tilde{\mathbf{q}}_{3}\right]=i \zeta_{k} \delta\left(\tilde{\mathbf{q}}_{1}+\tilde{\mathbf{q}}_{2}+\tilde{\mathbf{q}}_{3}\right) \times \\
& \left(\mu\left(\left(\mathbf{q}_{1} \cdot \mathbf{q}_{2}\right) \mathbf{q}_{3, \alpha}+\left(\mathbf{q}_{1} \cdot \mathbf{q}_{3}\right) \mathbf{q}_{2, \alpha}\right) \delta_{i_{2}, i_{3}} \delta_{\alpha, i_{1}}+\operatorname{perm} .(1,2,3)\right. \\
& \left.+\lambda\left(\mathbf{q}_{1} \cdot \mathbf{q}_{2}\right) \mathbf{q}_{3, \alpha} \delta_{i_{1}, i_{2}} \delta_{\alpha, i_{3}}+\operatorname{perm} \cdot(1,2,3)\right) \\
& \Gamma_{k, i_{1}, i_{2}, i_{3}, i_{4}}^{(4)}\left[\mathbf{r}_{k, f} ; \tilde{\mathbf{q}}_{1}, \tilde{\mathbf{q}}_{2}, \tilde{\mathbf{q}}_{3}, \tilde{\mathbf{q}}_{4}\right]=\delta\left(\tilde{\mathbf{q}}_{1}+\tilde{\mathbf{q}}_{2}+\tilde{\mathbf{q}}_{3}+\tilde{\mathbf{q}}_{4}\right) \times \\
& \left(\mu\left(\mathbf{q}_{1} \cdot \mathbf{q}_{2}\right)\left(\mathbf{q}_{3} \cdot \mathbf{q}_{4}\right)\left(\delta_{i_{1}, i_{4}} \delta_{i_{2}, i_{3}}+\delta_{i_{1}, i_{3}} \delta_{i_{2}, i_{4}}\right)+\text { perm. }(1,2,3,4)\right. \\
& \left.+\lambda\left(\mathbf{q}_{1} \cdot \mathbf{q}_{2}\right)\left(\mathbf{q}_{3} \cdot \mathbf{q}_{4}\right) \delta_{i_{1}, i_{2}} \delta_{i_{3}, i_{4}}+\text { perm. }(1,2,3,4)\right) \cdot(\mathrm{A} 8 \mathrm{~b})
\end{aligned}
$$

\section{Appendix B: Thresholds functions}

The RG flow equations Eqs.(32a)-(32c),(37a)-(37b) and (38) are expressed in terms of two families of threshold functions $l_{a b c}^{D}$ and $n_{a b c}^{D}$ defined in Eq.(33) and (39) that contain all the remnants of the loop integration of Eq.(20). In this Appendix, we write their dimensionless counterparts and compute them explicitly for the $\Theta$ cutoff.

\section{Dimensionless threshold functions}

We express the integrals Eq.(33) and Eq.(39) in terms of the dimensionless variables $y=q^{2} / k^{2}$ and $\bar{\omega}_{n}=$ $\omega_{n} /\left(\Delta_{k} k^{2}\right)$. We also introduce the variable: $Y=y^{2}+\bar{\omega}_{n}^{2}$ so that the cut-off function $R_{k}(\tilde{\mathbf{q}})$ entering in the expression (26) reads:

$$
R_{k}(\tilde{\mathbf{q}})=Z_{k} k^{4} Y \mathcal{R}(Y) .
$$

The two families of threshold functions $\bar{l}_{a b c}^{D}$ and $\bar{n}_{a b c}^{D}$ involve $\partial_{t} R_{k}(\tilde{\mathbf{q}})$. This last quantity can be made explicit thanks to the following relations (that take account of the fact that $\tilde{\eta}_{k}=0$ ):

$$
\begin{aligned}
& \partial_{t} \Delta_{k}=-\frac{1}{2} \eta_{k} \Delta_{k} \\
& \partial_{t} Y=-4 Y+\bar{\omega}_{n}^{2} \eta_{k} .
\end{aligned}
$$

This leads to, with the notations $\mathcal{R}=\mathcal{R}(Y), \mathcal{R}^{\prime}=$ $\mathcal{R}^{\prime}(Y)$ :

$$
\partial_{t} R_{k}(\tilde{\mathbf{q}})=-Z_{k} k^{4}\left(4 Y^{2} \mathcal{R}^{\prime}+\eta_{k}\left(y^{2} \mathcal{R}-\bar{\omega}_{n}^{2} Y \mathcal{R}^{\prime}\right)\right) .
$$

As seen in the expression (39) the threshold function family $n_{a b c}^{D}$ requires to compute additionally the quantity $\widehat{\partial}_{t} \partial_{q^{2}} P(\tilde{\mathbf{q}})$ where we recall that $P(\tilde{\mathbf{q}})=Z_{k} q^{4}+Z_{k}^{\tau} \omega_{n}^{2}+$ $R_{k}(\tilde{\mathbf{q}})$. Since the operator $\widehat{\partial}_{t}$ only acts on $R_{k}(\tilde{\mathbf{q}})$ one has the identity: $\widehat{\partial}_{t} \partial_{q^{2}} P(\tilde{\mathbf{q}})=\partial_{q^{2}} \partial_{t} R(\tilde{\mathbf{q}})$. This last quantity 
is easily computed from the expression Eq.(B3) and one gets:

$$
\begin{aligned}
\widehat{\partial}_{t} \partial_{q^{2}} P(\tilde{\mathbf{q}}) & =-2 Z_{k} k^{2} y\left(4 Y\left(2 \mathcal{R}^{\prime}+Y \mathcal{R}^{\prime \prime}\right)\right. \\
& \left.+\eta_{k}\left(\mathcal{R}+Y \mathcal{R}^{\prime}-2 \bar{\omega}_{n}^{2} \mathcal{R}^{\prime}-Y \bar{\omega}_{n}^{2} \mathcal{R}^{\prime \prime}\right)\right) .
\end{aligned}
$$

threshold functions $\bar{l}_{a b c}^{D}$ and $\bar{n}_{a b c}^{D}$ defined by:

Finally, we get the expressions of the dimensionless

with $\bar{P}_{i}(Y)=\bar{P}(Y)+\bar{m}_{i k}^{2} y, i=0,1,2$.

\section{Threshold functions with $\Theta$ cut-off}

The $\Theta$ cut-off is particularly useful if one wants to get simple analytical results. Using the expression Eq.(26), one has:

$$
\mathcal{R}(Y)=\frac{1-Y}{Y} \Theta(1-Y)
$$

where $\Theta$ is the Heaviside step function. The derivatives of $\mathcal{R}$ with respect to $Y$ are needed:

$\mathcal{R}^{\prime}(Y)=-\frac{1}{Y^{2}} \Theta(1-Y)-\frac{1-Y}{Y} \delta(1-Y)$

$\mathcal{R}^{\prime \prime}(Y)=\frac{2}{Y^{3}} \Theta(1-Y)+\frac{2}{Y^{2}} \delta(1-Y)-\frac{1-Y}{Y} \delta^{\prime}(1-Y)$.

Thanks to the properties of $\Theta$ and $\delta$ functions several terms simplify in the expressions of the threshold functions Eqs.(B6a) and (B6b). Also, the $\Theta$ function makes all remaining sums and integrals finite. We recall that the sums are cut at a maximal frequency given by

$$
\begin{aligned}
& n_{\max }=\left\lfloor 1 /\left(2 \pi \bar{T}_{k}\right)\right\rfloor: \\
& \bar{l}_{a b c}^{D}=\frac{\bar{T}_{k}}{2} \sum_{n=-n_{\max }}^{n_{\max }} \int_{0}^{\sqrt{1-\bar{\omega}_{n}^{2}}} d y y^{\frac{D}{2}-1} \frac{4+\eta_{k}\left(y^{2}-1\right)}{\left[1+\bar{m}_{1 k}^{2} y\right]^{b}\left[1+\bar{m}_{2 k}^{2} y\right]^{c}} \\
& \quad \times\left\{a+\frac{b}{1+\bar{m}_{1 k}^{2} y}+\frac{c}{1+\bar{m}_{2 k}^{2} y}\right\}
\end{aligned}
$$

$$
\begin{aligned}
\bar{n}_{a b c}^{D}= & -\bar{T}_{k} \sum_{n=-n_{\max }}^{n_{\max }}\left\{\int_{0}^{\sqrt{1-\bar{\omega}_{n}^{2}}} d y \frac{\eta_{k} y^{\frac{D+2}{2}}}{\left[1+\bar{m}_{1 k}^{2} y\right]^{b}\left[1+\bar{m}_{2 k}^{2} y\right]^{c}}\right. \\
& \left.-\frac{\left(4-\eta_{k} \bar{\omega}_{n}^{2}\right)\left(1-\bar{\omega}_{n}^{2}\right)^{\frac{D}{4}}}{2\left[1+\bar{m}_{1 k}^{2} \sqrt{1-\bar{\omega}_{n}^{2}}\right]^{b}\left[1+\bar{m}_{2 k}^{2} \sqrt{1-\bar{\omega}_{n}^{2}}\right]^{c}}\right\} .
\end{aligned}
$$

\section{Flat phase threshold functions}

In the flat phase, only massless threshold functions $\bar{l}_{a 00}^{D}$ and $\bar{n}_{a 00}^{D}$ remain for which the $y$-integrals in Eq.(B9a) and (B9b) can be computed explicitly and yield:

$$
\begin{aligned}
& \bar{l}_{a 00}^{D}=\bar{T}_{k} \sum_{n=-n_{\max }}^{n_{\max }}\left[4 \frac{a}{D}\left(1-\bar{\omega}_{n}^{2}\right)^{\frac{D}{4}}-\eta_{k} \frac{a\left(4+D \bar{\omega}_{n}^{2}\right)}{D(D+4)}\left(1-\bar{\omega}_{n}^{2}\right)^{\frac{D}{4}}\right] \\
& \bar{n}_{a 00}^{D}=\bar{T}_{k} \sum_{n=-n_{\max }}^{n_{\max }}\left[2\left(1-\bar{\omega}_{n}^{2}\right)^{\frac{D}{4}}-\eta_{k} \frac{4+D \bar{\omega}_{n}^{2}}{2(D+4)}\left(1-\bar{\omega}_{n}^{2}\right)^{\frac{D}{4}}\right] .
\end{aligned}
$$

As long as $2 \pi \bar{T}_{k}<1, n_{\max }>0$ and the frequency sum brings several contributions. 


\section{a. Vanishing temperature limit}

When $\bar{T}_{k} \rightarrow 0$, one recognises a Riemann integral:

$$
\bar{T}_{k} \sum_{n=-n_{\max }}^{n_{\max }} \underset{\bar{T}_{k} \rightarrow 0}{\longrightarrow} \int_{-1}^{1} \frac{d \bar{\omega}}{2 \pi} .
$$

The threshold functions now reads:

$$
\left\{\begin{array}{l}
\bar{l}_{a 00}^{D}=\frac{a}{2 \sqrt{\pi}} \frac{\Gamma\left[\frac{D}{4}\right]}{\Gamma\left[\frac{6+D}{4}\right]}-\eta_{k} \frac{3 a}{16 \sqrt{\pi}} \frac{\Gamma\left[\frac{D}{4}\right]}{\Gamma\left[\frac{10+D}{4}\right]} \\
\bar{n}_{a 00}^{D}=\frac{1}{\sqrt{\pi}} \frac{\Gamma\left[\frac{4+D}{4}\right]}{\Gamma\left[\frac{6+D}{4}\right]}-\eta_{k} \frac{3}{8 \sqrt{\pi}} \frac{\Gamma\left[\frac{4+D}{4}\right]}{\Gamma\left[\frac{10+D}{4}\right]} .
\end{array}\right.
$$

These expressions are valid only at $\bar{T}_{k}=0$ strictly speaking. However they still provide a very good approximation to the value of the threshold functions in the whole region $\bar{T}_{k} \ll 1$.

\section{b. High temperature limit}

At high-temperatures, the only remaining term in the frequency sum corresponds to $\bar{\omega}_{0}=0$. The threshold functions become:

$$
\left\{\begin{array}{l}
\bar{l}_{a 00}^{D}=\bar{T}_{k}\left[\frac{4 a}{D}-\eta_{k} \frac{4 a}{D(D+4)}\right] \\
\bar{n}_{a 00}^{D}=\bar{T}_{k}\left[2-\eta_{k} \frac{2}{D+4}\right]
\end{array}\right.
$$

The $\bar{T}_{k}$ factor in the flow equations is combined with the coupling constants to give back their value in the classical theory.
[1] K. S. Novoselov, A. K. Geim, S. V. Morozov, D. Jiang, Y. Zhang, S. V. Dubonos, I. V. Gregorieva, and A. A. Firsov, Science 306, 666 (2004).

[2] K. S. Novoselov, A. K. Geim, S. V. Morozov, D. Jiang, M. I. Katsnelson, I. V. Gregorieva, S. V. Dubonos, and A. A. Firsov, Nature 438, 197 (2005).

[3] M. I. Katsnelson, Graphene: Carbon in Two dimensions (Cambridge University Press, Cambridge, 2012).

[4] A. H. Castro Neto, F. Guinea, N. M. R. Peres, K. S. Novoselov and A. K. Geim, Rev. Mod. Phys. 81, 109 (2009).

[5] M. Katsnelson and A. K. Geim, Phil. Trans. R. Soc. A 366, 195 (2008).

[6] E. Mariani and F. von Oppen, Phys. Rev. Lett. 100, 076801 (2008).

[7] F. Guinea, B. Horovitz and P. Le Doussal, Phys. Rev. B 77, 205421 (2008).

[8] E. A. Kim and A. C. Neto, Europhys. Lett. 84, 57007 (2008).

[9] M. A. H. Vozmediano, M. I. Katsnelson, and F. Guinea, Phys. Reports 496, 109 (2010).

[10] E. V. Castro, H. Ochoa, M. I. Katsnelson, R. V. Gorbachev, D. C. Elias, K. S. Novoselov, A. K. Geim, and F. Guinea, Phys. Rev. Lett. 105, 266601 (2010).

[11] D. Gazit, Phys. Rev. B 80, 161406(R) (2009).

[12] P. San-José, J. González and F. Guinea, Phys. Rev. Lett. 106, 045502 (2011).

[13] F. Guinea, P. Le Doussal, and K. J. Wiese, Phys. Rev. B 89, 125428 (2014).

[14] J. González, Phys. Rev. B 90, 165402 (2014).

[15] R. Roldán, A. Castellanos-Gomez, E. Cappelluti and F. Guinea, J. Phys. Condens. Matter 27, 313201 (2015).

[16] B. Amorim, A. Cortijo, F. de Juan, A. G. Grushin, F. Guinea, A. Guttirez-Rubio, H. Ochoa, V. Parente, R. Roldán, P. San-José, J. Schiefele, M. Sturla and M. A. H. Vozmediano, Physics Reports 617, 1 (2016).

[17] D. R. Nelson, T. Piran, and S. Weinberg, eds., Proceed- ings of the Fifth Jerusalem Winter School for Theoretical Physics (World Scientific, Singapore, 2004), 2nd ed.

[18] D. R. Nelson and L. Peliti, J. Phys. (Paris) 48, 1085 (1987).

[19] J. A. Aronovitz and T. C. Lubensky, Phys. Rev. Lett. 60, 2634 (1988).

[20] E. Guitter, F. David, S. Leibler, and L. Peliti, J. Phys. (Paris) 50, 1787 (1989).

[21] J. A. Aronovitz, L. Golubovic, and T. C. Lubensky, J. Phys. (Paris) 50, 609 (1989).

[22] P. Le Doussal and L. Radzihovsky, Phys. Rev. lett. 69, 1209 (1992).

[23] M. Katsnelson and A. Fasolino, Acc. Chem. Res. 46, 97 (2013).

[24] B. Amorim, R. Roldán, E. Cappelluti, A. Fasolino, F. Guinea, and M. I. Katsnelson, Phys. Rev. B 89, 224307 (2014).

[25] A. L. C. Da Silva, L. Cândido, J. N. Teixeira Rabelo, G.Q. Hai, and F. M. Peeters, Europhys. Lett. 107, 56004 (2014).

[26] D. Gazit, Phys. Rev. E 80, 041117 (2009).

[27] K. V. Zakharchenko, R. Roldán, A. Fasolino and M. I. Katsnelson, Phys. Rev. B 82, 125435 (2010).

[28] E.I. Kats and V.V. Lebedev, Phys. Rev B 89, 125433 (2014).

[29] E.I. Kats and V.V. Lebedev, Phys. Rev B 90, 176301 (2014).

[30] B. Amorim, R. Roldán, E. Cappelluti, A. Fasolino, F. Guinea, and M. I. Katsnelson, Phys. Rev. B 90, 176302 (2014).

[31] C. Wetterich, Z. Phys. C 57, 451 (1993).

[32] J. Berges, N. Tetradis, and C. Wetterich, Phys. Rep. 363, 223 (2002).

[33] B. Delamotte, D. Mouhanna, and M. Tissier, Phys. Rev. B 69, 134413 (2004).

[34] J. Pawlowski, Annals Phys. 322, 2831 (2007).

[35] P. Kopietz, L. Bartosch and F. Schutz, Introduction to 
the Functional Renormalization Group (Springer, Berlin, 2010).

[36] H. Gies, Lect. Notes Phys. 852, 287 (2012).

[37] B. Delamotte, Lect. Notes Phys. 852, 49 (2012).

[38] O. Rosten, Phys. Rep. 511, 177 (2012).

[39] S. Nagy, Annals Phys. 350, 310 (2014).

[40] J.-P. Kownacki and D. Mouhanna, Phys. Rev. E 79, 040101 (2009).

[41] F. L. Braghin and N. Hasselmann, Phys. Rev. B 82, 035407 (2010).

[42] K. Essafi, J.-P. Kownacki, and D. Mouhanna, Phys. Rev. Lett. 106, 128102 (2011).

[43] N. Hasselmann and F.L. Braghin, Phys. Rev. E 83, 031137 (2011).

[44] K. Essafi, J.-P. Kownacki, and D. Mouhanna, Phys. Rev. E 89, 042101 (2014).

[45] A. Altland and B. D. Simons, Condensed Matter Field Theory (Cambridge University Press, Cambridge, 2010), 2nd ed.

[46] D. Litim, Phys. Lett. B 486, 92 (2000).

[47] C. Wetterich, Phys. Lett. B 301, 90 (1993).

[48] J.-P. Blaizot, R. Méndez-Galain, and N. Wschebor, Phys. Lett. B 632, 571 (2006).

[49] J.-P. Blaizot, R. Méndez-Galain, and N. Wschebor, Phys. Rev. E 74, 051116 (2006).

[50] J.-P. Blaizot, R. Méndez-Galain, and N. Wschebor, Phys. Rev. E 74, 051117 (2006).

[51] L. Canet, B. Delamotte, D. Mouhanna, and J. Vidal, Phys. Rev. B 68, 064421 (2003).

[52] O. Coquand, J.-P. Kownacki, and D. Mouhanna, unpublished (2016).

[53] D. Litim, Phys. Rev. D 64, 105007 (2001).

[54] D. Litim, Int. J. Mod. Phys. A 16, 2081 (2001).

[55] D. F. Litim, Nucl. Phys. B 631, 128 (2002).
[56] L. Canet, B. Delamotte, D. Mouhanna, and J. Vidal, Phys. Rev. D 67, 065004 (2003).

[57] I. Nandori, JHEP 1304, 150 (2013).

[58] I. G. Márián, U. D. Jentschura, and I. Nándori, J. Phys. G: Nucl. Part. Phys. 41, 055001 (2014).

[59] S. Chakravarty, B. I. Halperin, and D. R. Nelson, Phys. Rev. B 39, 2344 (1989).

[60] F. David and E. Guitter, Europhys. Lett. 5, 709 (1988).

[61] P. Le Doussal and K. J. Wiese, Phys. Rev. B 68, 174202 (2003).

[62] A. Rancon, O. Kodio, N. Dupuis, and P. Lecheminant, Phys. Rev. E 88, 012113 (2013).

[63] Cut-off functions $R_{k}(\mathbf{q})$ that effectively diverge in the limit $k \rightarrow \Lambda$, and thus that lead rigorously to a $\delta(\mathbf{R}-\mathbf{r})$ constraint, can be easily constructed.

[64] The leading order - called local potential approximation would consist to fix the field-renormalizations $Z_{k}$ and $Z_{k}^{\tau}$ to 1 leading to vanishing anomalous dimensions.

[65] See for instance [32] and references therein for general considerations about the NPRG approach to quantum systems and, for instance, [62] for an approach of the quantum relativistic $O(N)$ case, close in spirit to the present one.

[66] Another form of cut-off would impose the same kind of constraints but softened.

[67] We recall that the infra-red behaviour corresponds to $k \rightarrow 0$ and thus $t \rightarrow-\infty$.

[68] In the following equation the rescaling has not been made.

[69] The coefficient $64 \pi / 21$ here replaces the coefficient $64 \pi / 3$ in [13].

[70] In the quantum case, $D=2$ being the upper critical dimension there is no distinction between $\mathcal{Y}_{k}$ and $\overline{\mathcal{Y}}_{k}$. 